\title{
FULL-POWER TEST OF A STRING OF MAGNETS COMPRISING A HALF-CELL OF THE SUPERCONDUCTING SUPER COLLIDER
}

\author{
W. BURGETT, M. CHRISTIANSON, R. COOMBES, \\ T. DOMBECK, J. GANNON, D. HAENNI, P. KRAUSHAAR, \\ M. LEVIN, M. McASHAN, A. McINTURFF, G. MULHOLLAND, \\ D. MURRAY, W. ROBINSON, T. SAVORD, R. SMELLIE, \\ F. SPINOS, G. TOOL, J. WEISEND II, and J. ZATOPEK \\ Superconducting Super Collider Laboratory, 2550 Beckleymeade Ave., \\ Dallas, Texas 75237, USA
}

(Received 25 October 1992; in final form 30 July 1993)

\begin{abstract}
In this paper we describe the full-powered operation of a string of industrially-fabricated magnets comprising a half-cell of the Superconducting Super Collider (SSC). The completion of these tests marks the first successful operation of a major SSC sub-system. The five $15 \mathrm{~m}$ long dipole magnets in the string had an aperture of $50 \mathrm{~mm}$ and the single $5 \mathrm{~m}$ long quadrupole had an aperture of $40 \mathrm{~mm}$. Power and cryogenic connections were made to the string through spool pieces that are prototypes for SSC components. The string was cooled to cryogenic temperatures in early July 1992, and power tests were performed at progressively higher currents up to the nominal SSC operating point (above $6500 \mathrm{~A}$ ) achieved in mid-August. In this paper we report on the electrical and cryogenic performance of the string components and the quench protection system during these initial tests.
\end{abstract}

KEY WORDS: Magnets, Magnets: Superconducting, Refrigeration

\section{INTRODUCTION}

The Accelerator System String Test (ASST) was a congressionally mandated milestone for the Superconducting Super Collider Laboratory (SSCL) to demonstrate powered operation of a half-cell (five dipoles, one quadrupole, and spool pieces) of industrially fabricated collider magnets. Successful completion of the ASST was required before magnet mass production or tunnel boring could begin. The construction of the ASST buildings and installation of critical equipment was completed in a little over one year in a rural area of Texas rangeland that was the first SSCL site to be developed. The installation of the cryogenic refrigerator began in March 1992, and commissioning occurred in April. Magnet and spool piece installation in the string took place over the period from February to early June. Installation and commissioning of the power supply, quench protection system, Personnel Access Safety System (PASS), and control 
equipment was completed in late June. By the beginning of July, the string had been cooled to cryogenic temperatures, the final safety reviews had taken place, and the final stage of commissioning the magnet quench protection system leading to the power tests of the string had begun. Power tests were performed at progressively higher currents over a three-week period leading to the test at the nominal SSC operating point of $6500 \mathrm{~A}$ in mid-August.

The half-cell of magnets comprising the string is the basic subunit of the SSC collider lattice. A general description of its cryogenic and electrical operation is discussed in Reference 1. The ASST string was designed to be a prototype half-cell for the collider in order to test the general features of magnet interconnection, determine cryogenic heat loads under various operating conditions, and demonstrate the safe operation of the string under normal powered conditions and during magnet quenches. The dipole magnets were fabricated by the General Dynamics Corporation ${ }^{2}$ with tooling in place at Fermi National Accelerator Laboratory (FNAL). The quadrupole magnet was fabricated at Lawrence Berkeley Laboratory (LBL) and placed in its cryostat at the SSCL.

The string was assembled in the western half of a $200-\mathrm{m}$-long building, with associated electronics installed in a niche area on the south side of the string and in a controls trailer outside the enclosure. The refrigerator was installed in a building attached to the enclosure, with compressor and storage dewars mounted outside on a concrete pad. The power supply and its associated equipment were installed in a room about $80 \mathrm{~m}$ away from the string. The basic parameters for the ASST components are summarized in Table 1.

The installation of the string components, the vacuum pump down of the magnet cryostats and the commissioning of the string subsystems are discussed in this paper. The operation of the string both electrically and cryogenically is described, and the performance of the string components during the power tests is presented. Although there were no natural quenches of the magnets during these tests, results on the operation of the quench protection system during induced quenches are presented. Preliminary results from heat leak measurements and quench propagation in the string are also presented.

\section{THE STRING COMPONENTS AND PRINCIPLES OF OPERATION}

\subsection{Dipole and Quadrupole Magnets}

The SSC dipole magnets used in the ASST have four quarter coils wound in a $\cos \theta$ distribution around a beam aperture of $50 \mathrm{~mm}$. The two inner coils arranged above and below the beam tube are designated as UI and LI respectively, while the two coils outside the inner coils are designated UO and LO. The coils are encased in stainless steel collars and pre-stressed in excess of $50,000 \mathrm{kPa}$. This assembly is encased in the iron yoke of the magnet which is then enclosed in a stainless steel vessel through which single-phase, super-critical helium flows at a nominal $4.35 \mathrm{~K}$ at a pressure of 4 bars. This final assembly, called the magnet cold mass, is surrounded by a thermal shield 
TABLE 1: Parameters of the ASST Magnets and Equipment

\begin{tabular}{|c|c|}
\hline Dipole Magnet & \\
\hline Inductance $(\mathrm{mH})$ & 75 \\
\hline Aperture & $50 \mathrm{~mm}$ \\
\hline Length & $15 \mathrm{~m}$ \\
\hline Operating Field & $6.6 \mathrm{~T}$ \\
\hline Nominal Operating Current & $6506 \mathrm{~A}$ \\
\hline Stored Energy & $1.6 \mathrm{MJ}$ \\
\hline Quench Heater Energy & $3.5 \mathrm{~kJ} /$ heater pair \\
\hline MIITs Limit on Inner Coils [3] & 20 \\
\hline MIITs Limit on Quter Coils [3] & 15 \\
\hline Mass & 13.6 metric tons \\
\hline Anticipated Cold Mass Heat Leak & $363 \mathrm{~mW}$ \\
\hline \multicolumn{2}{|l|}{ Quadrupole Magnet } \\
\hline Inductance $(\mathrm{mH})$ & 7 \\
\hline Aperture & $40 \mathrm{~mm}$ \\
\hline Length & $5.2 \mathrm{~m}$ \\
\hline Operating Field & $206 \mathrm{~T}$ \\
\hline Nominal Operating Current & $6506 \mathrm{~A}$ \\
\hline Stored Energy & $0.15 \mathrm{MJ}$ \\
\hline Quench Heater Energy & $1.2 \mathrm{~kJ} /$ heater pair \\
\hline MIITs Limit on Inner Coils [3] & 12 \\
\hline MIITs Limit on Outer Coils [3] & 10 \\
\hline Mass & 1.32 metric tons \\
\hline \multicolumn{2}{|l|}{ Spool Pieces } \\
\hline Mass Flow on Power Leads & $2 \mathrm{~g} / \mathrm{s}$ \\
\hline \multicolumn{2}{|l|}{ Refrigerator } \\
\hline Mass Flow & $54 \mathrm{~g} / \mathrm{s}$ \\
\hline Liquefaction Rate & $150 \mathrm{l} / \mathrm{hr}$ \\
\hline \multicolumn{2}{|l|}{ Power Supply } \\
\hline Maximum Voltage & $10 \mathrm{~V}$ \\
\hline Maximum Current & $8000 \mathrm{~A}$ \\
\hline Maximum Ramp Rate & $6 \mathrm{~A} / \mathrm{s}$ \\
\hline Dump Time Constant-all 6 Magnets & $24 \mathrm{~s}$ \\
\hline Dump Time Constant-3 Dipole Magnets & $12 \mathrm{~s}$ \\
\hline
\end{tabular}


cooled by $20 \mathrm{~K}$ helium gas, which in turn is surrounded by an $80 \mathrm{~K}$ liquid nitrogen thermal shield. The entire magnet assembly is supported within an insulating vacuum vessel. Representative cross sections for the SSC magnets can be found in References 1 and 3.

Each magnet has four strip heaters, two upper and two lower, around the outside edges of the UO and LO coils. After a quench is detected, the strip heaters are activated to increase the normal zone in the superconducting cable in order to uniformly dissipate heat generated during the quench, thus reducing the number of MIIT generated in the magnet. (MIITs are the integral of $I^{2} \mathrm{dt}$ during the time that the magnet is normal, measured in units of $10^{6}$. See reference 4.) The strip heaters are connected in pairs on two separate series circuits for each magnet, and are activated independently by two capacitor banks per magnet. This provides a means of tuning the amount of energy delivered to each magnet heater by adjusting the capacitance and voltage. The redundant set of heaters provides additional protection for each magnet in case of an equipment failure. To detect quenches, each magnet is instrumented with an array of voltage taps soldered onto each quarter coil. During the sequence of magnet quenching and subsequent heater activation, the voltage taps are used to measure the voltages generated in the string.

Before installation in the string, each dipole magnet was individually power tested at FNAL's Magnet Test Facility. ${ }^{5}$ Each magnet showed good quench behavior, reaching a plateau above the nominal operating current of $6500 \mathrm{~A}$ (corresponding to a field of $6.6 \mathrm{~T}$ ) at the nominal operating temperature of $4.35 \mathrm{~K}$. Three of the dipole magnets experienced one "training" quench below $6500 \mathrm{~A}$. After this initial quench, each reached the short sample limit of the superconducting cable near $7300 \mathrm{~A}$ and did not degrade below the operating current even after subsequent thermal cycling of the magnet. The other two dipole magnets reached short sample current limits without training. The current-carrying margin for the dipole magnets was approximately $12 \%$; this implied that the magnets could be operated at temperatures up to $4.9 \mathrm{~K}$ at 6500 A. Another result of the single magnet tests was the consistency of the magnet quench performance from one dipole magnet to another.

One unexpected feature of the dipole magnets was the rapid degradation of quench performance with increasing ramp rate (di/dt). This appears to be caused by differing strand-to-strand resistance in each case, though the exact cause is still under investigation. ${ }^{5}$ The degradation of quench performance below the nominal current level of $6500 \mathrm{~A}$ for each magnet was present only at ramp rates far in excess of the 4 $\mathrm{A} / \mathrm{s}$ rate expected for collider operation. Therefore, this behavior was not expected to have an effect in the string operation.

The ASST quadrupole magnet has an aperture of $40 \mathrm{~mm}$ with the superconductor wound in eight coils arranged in four quadrants. ${ }^{1}$ It was fabricated with 30 -strand cable, which has a lower limit on MIITs than the 37-strand cable used in the 50-mm aperture dipole magnets. For this reason it was necessary to provide an additional bypass diode to conduct current from the dipole magnets around the quadrupole magnet when the quadrupole magnet experienced a quench (see the discussion in Section 2.4). The quadrupole magnet is equipped with eight quench heaters arranged around the outer coils in two series circuits, similarly to the dipole magnets. 
The quadrupole magnet cold mass was designed, manufactured, and tested at LBL. The cold mass was placed in its cryostat at the SSC Laboratory and subsequently power tested on a test stand at Brookhaven National Laboratory (BNL). It showed a substantial number of "training" quenches. This magnet experienced additional "training" quenches upon subsequent thermal cycle. ${ }^{6}$

Using the designations in Reference 5, the five dipole magnets in the ASST, in order of their installation, were DCA313, DCA314, DCA319, DCA315, and DCA316. The quadrupole magnet was designated as QC403. These magnets were arranged such that the first three dipoles were energized from one bus (lower), while the last two dipoles and the quadrupole were energized from a separate bus (upper). This arrangement permitted the magnets to be separated into two triplets, each on its own power bus, as discussed in Section 2.4 on Power Equipment and String Electrical Operation. This was an important feature of the half-cell operation during magnet quenches, where, in principle, only one-half of the magnets had to be quenched at any time. Having only half the inductance on one bus reduced both the heat loads on the refrigerator system and the voltages generated in the magnets.

In addition to the voltage taps, each magnet was equipped with pressure and temperature sensors in the cold mass region and in each of the thermal shields in the interconnect region. These sensors were continuously monitored throughout the run, permitting measurements to be made at various points along the length of the string under different operating conditions.

\subsection{Spool Pieces}

Three spool pieces were installed in the ASST, one-half of a standard collider feed spool (HSPRF), a standard collider recooler spool (SPR), and a special end spool (HSPRE) to terminate the string. ${ }^{3}$ These prototypes of collider spools were designed at the SSCL and each was manufactured by a different company. ${ }^{7}$ Cryogenic fluids were provided to the magnet string by a refrigeration system connected to the HSPRF at the lead end of the string. Liquid helium flowed serially through the magnet cold masses, then returned back up the string to the refrigeration system through separate piping installed in the magnets. Liquid nitrogen flowed through the $80 \mathrm{~K}$ shield and was expelled to the atmosphere at the end of the string. In the ASST the reversal of the cryogenic flow was accomplished in the HSPRE placed at the end of the string. The ASST also contained an SPR with a recooler that permits heat extraction in a long string of magnets, though the feature was not used in this string test. Ultimately in the collider the SPR would also contain corrector magnets and beam position monitors, but these were not installed for the ASST. The SPR also contained a vacuum break $^{3}$ composed of a re-entrant-shaped piece of stainless steel extending from the cold mass to the outer vacuum jacket and tied to the heat shields at the appropriate radii.

Each spool piece was equipped with pressure relief valves on the cold mass as well as on each cryogenic line at both ends of the string to protect the magnet system from over-pressuring. All three spool pieces were equipped with quench valves that could be pneumatically activated by an electrical signal sent from the quench protection 
system when a quench was detected. This allowed warm gas generated in the cold mass to be exhausted into the $20 \mathrm{~K}$ shield line. Although there were quench valves at both ends of the string, only the one on the HSPRE was used in the initial power tests.

The spools also provided the electrical connections to the string for power and for the instrumentation installed in the string components. Power leads designed with a copper bus attached to superconducting cable ${ }^{8}$ were installed in the HSPRF and HSPRE, and were cooled by counter-flow gaseous helium returned to the refrigeration system. The gas flow rate was controlled to maintain a superconducting state in the cold-end connection of the lead. Instrumentation connections were made through multi-pin connectors mounted on each spool piece with the wiring to the cryogenic lines encased in coils of stainless steel tubing that were attached to the intermediate shields, which acted as heat sinks.

The SPR contains a quench stopper with a set of attached bypass leads that play an important role in the collider design. Its purpose is to prevent a quench from propagating thermally through the power bus along the string of magnets. ${ }^{1}$ The details of the quench stopper design have previously been given. ${ }^{3}$ It consists of two copper ribbed cages that act as heat sinks for the superconducting cable attached to them. There are separate lengths of superconductor for each of the two power buses for the magnets. The bypass leads are made of stainless steel rod approximately $4 \mathrm{~cm}$ in diameter and attached to the copper ribs that carry off current if the magnets on one or the other side of the quench stopper turn normal. The stainless steel rods are about $2 \mathrm{~m}$ in length and coiled before exiting the vacuum outer jacket. In order to expedite quench recovery, the bypass leads were cooled by gaseous helium flow. If a quench had occurred and substantial current had flowed through the bypass leads, thus raising their temperature, gaseous helium would have been allowed to flow out the leads long enough to return the leads to operating temperature in 30 minutes. In the ASST, the SPR leads provided a means to connect a bypass diode around the quadrupole magnet to isolate it from the dipole magnets on the same power bus during a quench. Due to its complexity, the SPR was tested cryogenically and electrically at BNL prior to installation in the string.

\subsection{Refrigerator Equipment and String Cryogenic Operation}

The refrigerator for the power testing phase of the ASST was built by Cryogenics Consultants, Inc. ${ }^{9}$ and provided $525 \mathrm{~W}$ at $4.5 \mathrm{~K}$ and a mass flow of $54 \mathrm{~g} / \mathrm{s}$. In comparison, one of the ten final sector refrigerators for the SSC will provide $6.7 \mathrm{~kW}$ at $4.35 \mathrm{~K}$ at a mass flow of $100 \mathrm{~g} / \mathrm{s}^{1}{ }^{1}$ The ASST refrigerator was capable of a liquefaction rate of $135 \mathrm{1} / \mathrm{hr}$. There were intermediate modes of operation that provided both refrigeration and liquefaction at reduced rates. The refrigerator system had a 500-hp, 70-g/s, 16-bar screw compressor, as well as a cold box with warm and cold, single-piston reciprocating expanders. There was also an auxiliary cold box with a precooler, a subcooler, and a cold compressor for lower temperature operation. A $41.6 \mathrm{kl}$ liquid nitrogen dewar supplied the refrigerator nitrogen precooling heat 


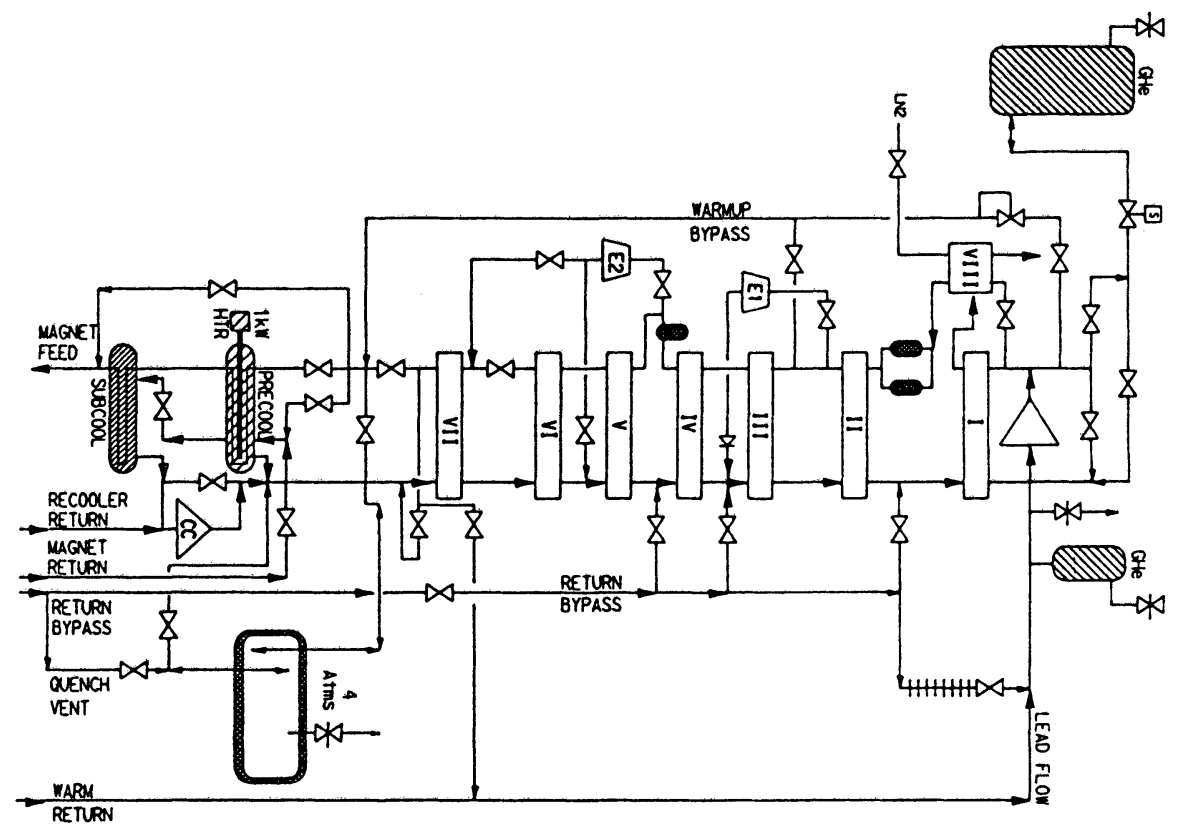

FIGURE 1: The schematic diagram of refrigerator operation. There were seven stages of heat exchangers in the refrigerator cold box, along with an eighth allowing precooling by heat exchange with liquid nitrogen. The precooler and a subcooler were mounted on the distribution box and permitted lower temperature operation.

exchanger and provided for the $80 \mathrm{~K}$ magnet shield flow. The nitrogen vapor was vented to the atmosphere. Gaseous helium was stored in an $80 \mathrm{kl}, 17$ bar tank. Liquid helium was stored in a $5 \mathrm{kl}, 4$ bar dewar. This dewar was filled by the refrigerator in the liquefaction mode. It also served as a cold storage reservoir for expelled quench gas. A schematic diagram of the refrigerator system is shown in Figure 1.

To maintain optimum cooling during normal string cooling operations, $54 \mathrm{~g} / \mathrm{s}$ flow was sent through the magnet cold masses, and about $0.5 \mathrm{~g} / \mathrm{s}$ was dissipated in each of the four main power leads in the HSPRF and HSPRE. During power operations the flow to the power leads was increased by as much as $10 \mathrm{~g} / \mathrm{s}$ in order to maintain sufficient cooling. At the end of the string most of the flow was returned to the refrigerator via the liquid return line, but about $2 \mathrm{~g} / \mathrm{s}$ flow was returned up the 20 $\mathrm{K}$ shield line to maintain its temperature.

When one or more magnets quenched, the helium in the magnet coils was rapidly expelled. The helium in the cold masses subsequently warmed to a maximum temperature near $30 \mathrm{~K}$, and the pressures rose to about 9 bar. The quench valve in the HSPRE spool opened to provide a path for the warm helium out of the cold mass and back to the refrigeration system dewar through the $20 \mathrm{~K}$ shield line. The refrigerator 


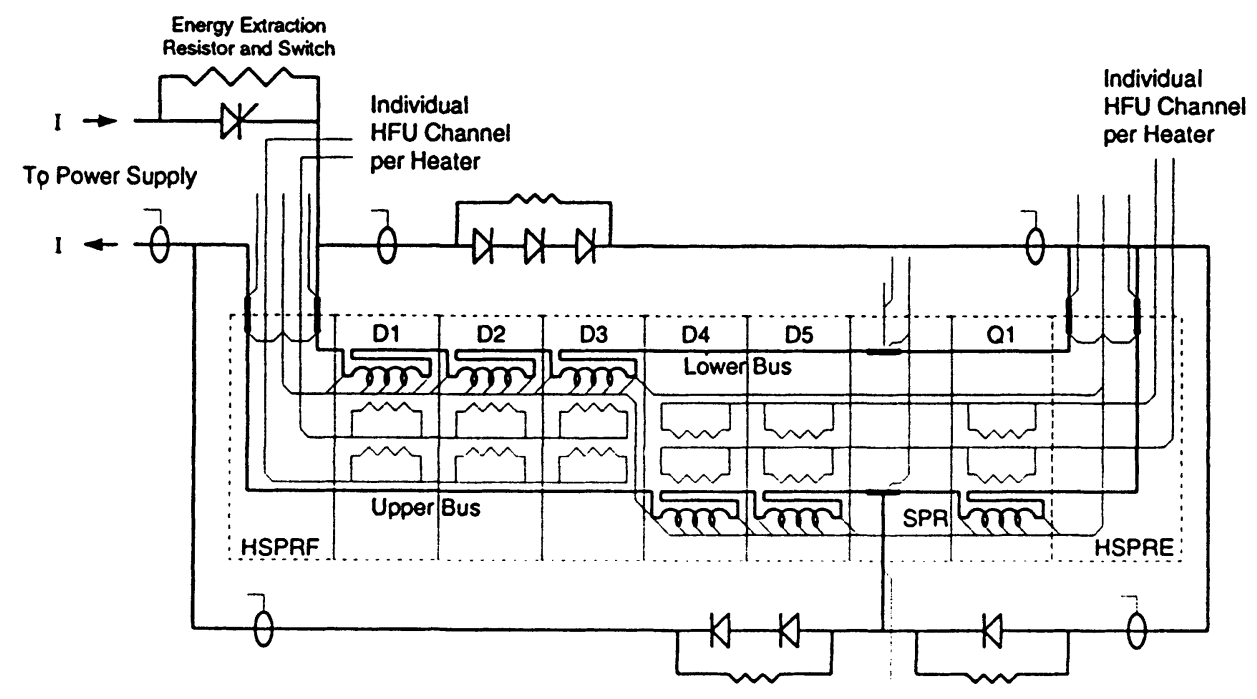

FIGURE 2: The ASST electrical circuit. The dipole magnets (D) and the quadrupole magnet (Q) are wired in a series circuit. The internal buses are wired in conjunction with bypass diodes to divide the circuit during quenches, as described in the text.

then recooled the string, liquefied the gas, and refilled the string after each quench. The helium system was scaled to be able to completely contain full power quenches, thus all the helium used in the refrigeration of the magnet string was recovered.

\subsection{Power Equipment and String Electrical Operation}

Power was provided to each magnet coil through internal buses that connected the half-cell of magnets in a series circuit as shown schematically in Figure 2. SSC collider operation requires that the magnet current be ramped up at a rate of $4 \mathrm{~A} / \mathrm{s}$ to a maximum current of $6500 \mathrm{~A}$. For the ASST, a ramping power supply capable of generating up to $8000 \mathrm{~A}$ at $40 \mathrm{~V}^{10}$ was connected to the string through an approximately $80 \mathrm{~m}$ long power bus consisting of ten high-current cables carrying current in one direction interspersed with ten cables carrying current the other way. The voltage drop across this length of power bus was about $4 \mathrm{~V}$. A high-power dump switch connected in parallel with a dump resistor was installed between the power supply and the power bus. During magnet quenches, the power supply was turned off and the dump switch opened to allow energy to be extracted from the string through the dump resistor. The power supply and silicon controlled rectifiers (SCRs) in the dump switch were watercooled using low conductivity water (LCW) supplied by a heat exchanger and purifier 
mounted on a separate skid. The dump resistor was mounted outside the power supply building and was air-cooled.

As mentioned above, the electrical wiring within the magnets was arranged such that the first three dipole magnets were isolated on one power bus, while the last two dipole and quadrupole magnets were isolated on the other power bus. Under normal superconducting operation, the string of magnets conducted power in a series circuit, but during a quench the string divided into two separate circuits. In Figure 2 the string is shown connected to bypass diodes through outside power buses. Under normal superconducting operation, there was insufficient forward-bias voltage across the diodes to allow conduction. During a magnet quench, only the diodes that were associated with the power bus containing the quenched magnets were turned on. Because of this arrangement, it was not necessary to quench all of the magnets in the string, but only those in the set where the initial quench was observed. The quench protection circuitry was designed to activate only the quench heaters in that set of quenching magnets. The current was shunted through the bypass diodes around the quenching triplet and continued to flow through the triplet of magnets that did not quench. During a quench, the power supply was turned off and the dump resistor circuit activated. The energy in the magnets that were still superconducting was extracted through the dump resistor and did not appear as heat in the refrigerator system. The dump resistance was set to provide a current decay in the superconducting magnet triplet with a time constant of $12 \mathrm{~s}$, whereas the decay constant for the quenching triplet was near $275 \mathrm{~ms}$.

Magnet quenches were detected by the voltage taps attached to each quarter coil of the magnets. Other voltage taps were provided at various locations on the power leads and power buses to detect quenches in all of the superconducting regions in the string. Quenches detected by monitoring the voltage taps activated the appropriate quench heaters in order to protect the string. The threshold for quench detection was set at $0.5 \mathrm{~V}$ for the magnets and power buses for currents above $50 \mathrm{~A}$. For currents below $50 \mathrm{~A}$ the thresholds were set at $2 \mathrm{~V}$ to avoid trips from transients in the circuit associated with turning on the power supply. The power buses were not designed to take a current decay with a $12 \mathrm{~s}$ time constant in the normal resistive state, so if a quench were detected in any of the power buses, all of the quench heaters in all of the magnets would be activated.

Each half of the string circuit was treated independently by the quench detection and protection system. Even though the initial quench was observed in one half or the other of the string and the appropriate quench heaters activated, it was still possible that the quench might thermally propagate to the other half of the string, or that an unrelated quench might occur. Therefore, the quench detection circuitry stayed active to protect the non-quenching side of the string even as the current was being reduced in those magnets.

\subsection{On-Line Control System and Data Acquisition}

The power operations described in the preceding section were controlled through programmable local processors interconnected through an optical fiber network to 
a controls trailer just outside the string enclosure building. The ramping and other functions for the power supply and dump switch control were performed by a local processor known as the Collider Excitation Controller and Regulator (CECAR) situated in an electronics rack near the power supply. The quench protection logic was performed by another processor called the Quench Protection Monitor (QPM) mounted near the heater firing capacitor banks in a niche area along the side of the magnet string. There was also a separate hard-wired system mounted in the niche to shut down the power supply and bring the dump resistor into the circuit if a fault occurred in the QPM logic. These processors were capable of maintaining a continuously updated circular log of the voltages and currents generated during the ramping of the current, along with any detected quenches. The rate of acquisition of this data was $720 \mathrm{~Hz}$. Because transient voltages generated during quenching could become large, it was necessary to confine them to the string enclosure and niche area. These voltages were monitored in the control trailer using isolation amplifiers.

The activation of the quench valves and the control of the gaseous helium flow for the power and bypass leads in the spool pieces was accomplished by a commercially available system known as TISTAR. ${ }^{11}$ This system had its own network using bi-axial cable and programmable logic controllers (PLC) mounted locally throughout the system. TISTAR also performed the control functions for the LCW skid, and monitored a subset of the temperature, pressure, and vacuum sensors installed in the string. In addition, the system maintained a continuous log of these measurements and provided alarms in the controls trailer if sensor readings drifted out of prescribed limits.

The temperatures, pressures, and voltages in the magnet and spool piece cold masses were also monitored by the on-line Research Instrumentation Data Acquisition System (RIDAS) at a sampling rate that could be varied from one to five minutes. This VXI-based system ${ }^{12}$ also monitored the temperatures and pressures in the $20 \mathrm{~K}$ and $80 \mathrm{~K}$ shields, as well as the vacuum in the cryostats and in the beam pipe. A transient digitizing system was used to collect high resolution data during a quench. High bandwidth (up to $2 \mathrm{kHz}$ ) thermal and pressure data were collected. Data were also collected at this rate from magnet voltage taps, current transductors, and accelerometers. In all, there were approximately 300 channels of data read out using seven 32-channel data loggers and stored on two redundant hard disks in a database using ORACLE. ${ }^{13}$ All of these programs ran on Sun workstations and HP360 computers. ${ }^{14}$

Many of the temperature and pressure sensors required special use, such as reversal of the currents applied to their four-wire probe circuits, to obtain highly accurate measurements. This allowed automatic subtraction of intrinsic noise and bias levels. ${ }^{15}$ This current reversal function, as well as signal amplification, was performed by the RIDAS system in specially prepared front-end modules. The on-line program stored the raw data, performed the subtractions, and applied calibration/conversion functions to obtain the engineering quantities for later analysis.

Data could be analyzed and plotted both on and off line by using the N!POWER ${ }^{16}$ and $\mathrm{XVGR}^{17}$ programs and accessing the database. In the case of on-line data, scrolling 
graphs were used to monitor trends in temperatures during the cool down. For example, algorithms were written to calculate the MIITs generated during quenches.

Access to the string area was controlled during power testing. The Personnel Access Safety System (PASS) was operated from the controls trailer, and consisted of monitored gates and doors isolating the magnet string. ${ }^{18}$ The PASS was activated by walking through the string enclosure and inserting keys into monitor boxes at specific locations. Only after the area was cleared and PASS activated could the power supply be turned on. The PASS also monitored the oxygen content in the refrigerator and string areas to detect any oxygen deficiency hazard (ODH). Alarms would have sounded and the building would have been cleared if a hazard had been detected.

\section{EQUIPMENT INSTALLATION AND COMMISSIONING}

\subsection{String Component Interconnections}

The magnet string was assembled in a $200 \mathrm{~m}$ long enclosure having a width of 4.3 $\mathrm{m}$ and the same curvature along its length as the SSC tunnel. The magnet handling equipment consisted of specially prepared dollies and electric carts. Hydraulic jacks and A- frame cranes were used to place the magnets on their stands. The magnets were then surveyed into position and aligned using bolts on the stands. This was also done for the spool pieces. Special care was taken to ensure that the bolts holding the end spools to the floor would withstand the lifting forces anticipated during magnet quenches. The motion of these components was monitored during pressure tests of the string to ensure that there would be no problem during the run.

The magnet interconnects were designed to provide quick and reliable connections for both the cryogenics and electrical lines from one component to the other. The details are given elsewhere. ${ }^{3}$ Many of the electrical lines to be connected were unique to the string test and will not be present in the collider. For example, the temperature and pressure instrumentation and many of the voltage taps were installed so that conditions within the string could be monitored closely during magnet quenching. To accommodate these additional components the end domes on the magnet cold masses were extended $30 \mathrm{~cm}$ on each end, and special instrumentation wire bundles were pulled through each magnet to allow the signals to be carried through the neighboring magnet, terminating at the spool piece connectors.

The electrical connections included special solder joints for the two superconductor power buses. A special fixture was prepared to hold the splice joint and apply the correct temperature to the solder. Four of the splice joints were instrumented with low-inductance voltage taps in order to measure the voltage drop (approximately $10^{-7}$ volts) when high current was applied to the magnets. After correcting for thermal effects, the joint resistances were measured at currents of up to $6000 \mathrm{~A}$, to be from 0.9 to $1.3 \times 10^{9} \Omega$ each. This was more than a factor of two below the resistance at 


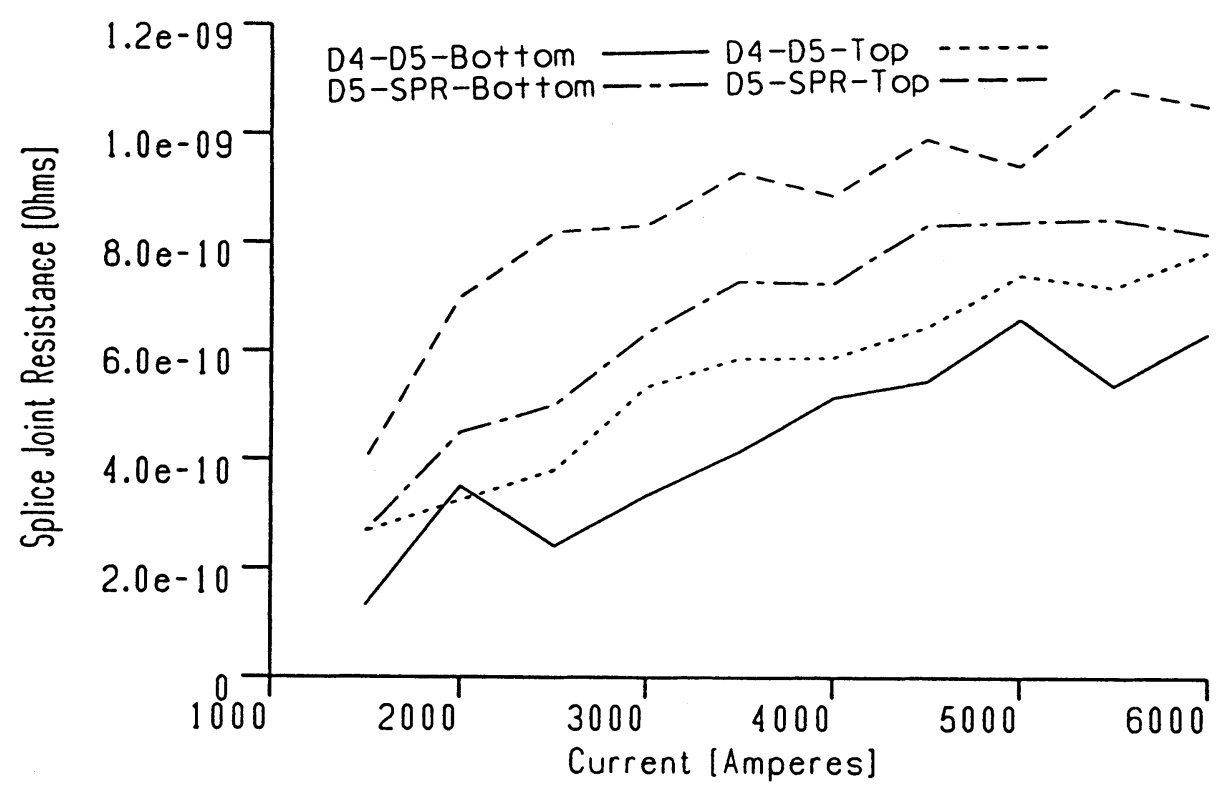

FIGURE 3: The results of resistance measurements made on the power bus splice joints connecting dipole magnets in the string, shown as a function of the current run in the magnets. These measurements have been corrected for temperature offsets. At low currents the amperage can be carried through the lead in the solder used in the joint connection, but it is expelled at higher currents. This accounts for the increase in resistance observed in the plot as the current increases. The worst resistance recorded is only a factor of two below that at which heat generated in the joint could make the bus quench.

which heating in the joint could make the superconducting cable prematurely resistive (see Figure 3).

The voltage taps and quench heater connections from one magnet to another were made by hand-soldering the wires together. The temperature and pressure sensors were interconnected by special wire harnesses with multi-pin connectors inside the magnet cold mass end domes. These harnesses were prepared ahead of time and jumpered from one magnet to another. Once the connections were finished, insulated, and restrained they were subjected to a "hipot" and continuity check.

The mechanical connections were made after the wiring was completed. Bellows connecting the cryogenic piping and beam pipe were welded manually, while the bellows connecting the cold masses were welded using an automatic device. Squirm protectors were placed over each bellows. The aluminum heat shields were completed in the interconnect with extensions screwed into place and covered with multi-layer insulation. The outer vacuum sleeves over the interconnect region were pulled hydraulically over large O-ring seals mounted concentrically on the ends of the magnet cryostats. 
After the entire string had been assembled, but before introducing helium into the system, final checks were made on the electrical integrity. A $1500 \mathrm{~V}$ hipot test to ground was made of all connections. One ampere of current was introduced into the power bus and the voltage drops were measured between the front end of the bus and each voltage tap in the string. This permitted a check, by the ordering and magnitude of the measured resistances, that we had properly identified each voltage tap to the appropriate connector pin.

\subsection{Cryostat Vacuum Pumpdown}

All of the interconnects were completed in early June, and vacuum leak checking commenced on the cryostat. Each interconnect outer sleeve was provided with a pump out port and leak detectors. A leak was detected and isolated to be at one particular bellows section in the interconnect between the fourth and fifth dipole magnets. The vacuum sleeve and heat shields were removed, the joint rewelded, the interconnect reassembled, and the string back under vacuum in only five hours.

The cryostats were pumped down using turbopumps. Due to the large quantity of water vapor trapped in the multi-layer insulation, the vacuum reached values near $10^{-3} \mathrm{~Pa}$ after three weeks of pumping. Eventually the string vacuum attained values better than $10^{-6} \mathrm{~Pa}$ after the cold mass was below $50 \mathrm{~K}$ and cryo-pumping began. The beam pipe was also in contact with the liquid helium in the cold mass region and attained a good vacuum. This was achieved with an ion pump mounted on the SPR.

\subsection{Pressure Test of the Cold Mass and Cryogenic Transfer Lines and System Purification}

The nominal helium pressure in the cold mass was 4 bars during normal string operations. From previous experience with magnet strings, single magnet test data, and calculations using models of SSC magnets, ${ }^{19}$ it was estimated that the cold mass would experience a maximum pressure spike near 10 bars during a high current quench of the magnets. To certify the string, a pressure test was performed at 20 bars on the cold mass, interconnect piping, and cryogenic transfer lines from the refrigerator. Helium was used as the working gas in order to also perform a high-sensitivity vacuum leak check between the cryogenic lines and the cryostat. Once certified at the working pressure, the pressure relief valves on the spool pieces were set to 13 bars as added protection for the magnet pressure vessels.

During the pressure test an unusual helium leak was observed in the SPR that only opened above a pressure of 4.6 bars. It then disappeared when the pressure was reduced. The leak appeared to be on the valve controlling the helium flow from the liquid return line to the recooler. The leak was small (about $10^{-9} \mathrm{cc} / \mathrm{s}$ ) and had been missed earlier because the manufacturer did his leak tests below 4.6 bars. Since the recooler was not being used in the early stages of the ASST, and because the cold mass operating pressure was below the point where the leak occurred, the cooldown of the magnets was continued. Additional turbopumps were provided near the point where the leak was observed in case the leak worsened. In fact, continual monitoring 


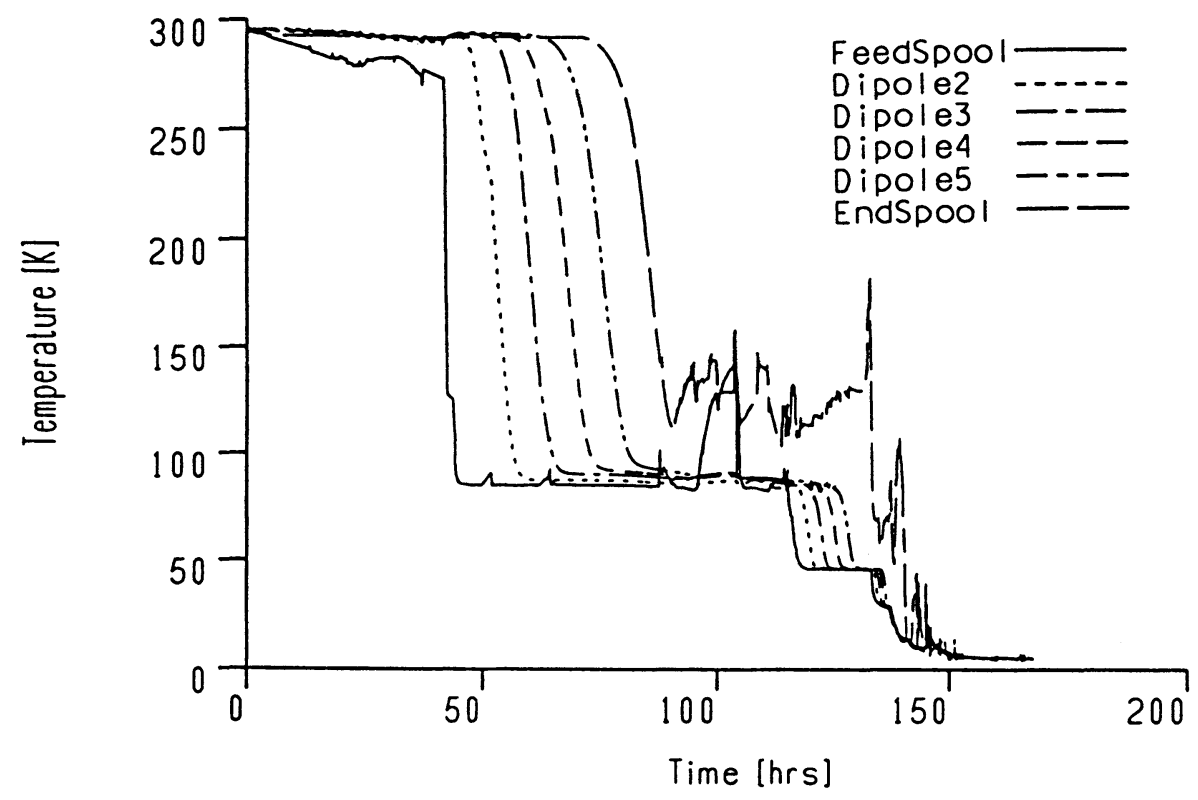

FIGURE 4: The string cool down progression. The refrigerator was operated in different modes to allow waves of helium at $80,50,20,10$ and $5 \mathrm{~K}$ to flow through the magnets. The temperatures are monitored in each magnet cold mass and show the serial cooling of each string component. The string was superconducting in $150 \mathrm{hrs}$.

using a residual gas analyzer (RGA) showed no evidence of the leak's reappearance throughout the experiment, even during magnet quenches.

The refrigerator system required the elimination of water vapor from the magnet cold masses to about $15 \mathrm{ppm}$ in order not to contaminate the heat exchangers. This was accomplished by purging the cold mass first with dry nitrogen and then with dry helium gas for up to three weeks before flowing cold helium through the system. We believe that this time could have been reduced significantly by circulating warm helium through the entire refrigerator magnet string system with a purifier and a molecular sieve in the circuit.

\section{STRING COOLDOWN}

\subsection{String Cooldown Data}

Figure 4 shows the time progression of the cool down of the ASST string of magnets. The cool down began with a wave of helium gas cooled to $80 \mathrm{~K}$ at the inlet side of the string. The progress of the wave through each magnet is evident from the plot. Once the string cooled below $100 \mathrm{~K}$, the expanders on the refrigerator were turned on, and the string was cooled progressively in $50 \mathrm{~K}, 20 \mathrm{~K}, 10 \mathrm{~K}$ and $5 \mathrm{~K}$ waves. After the 


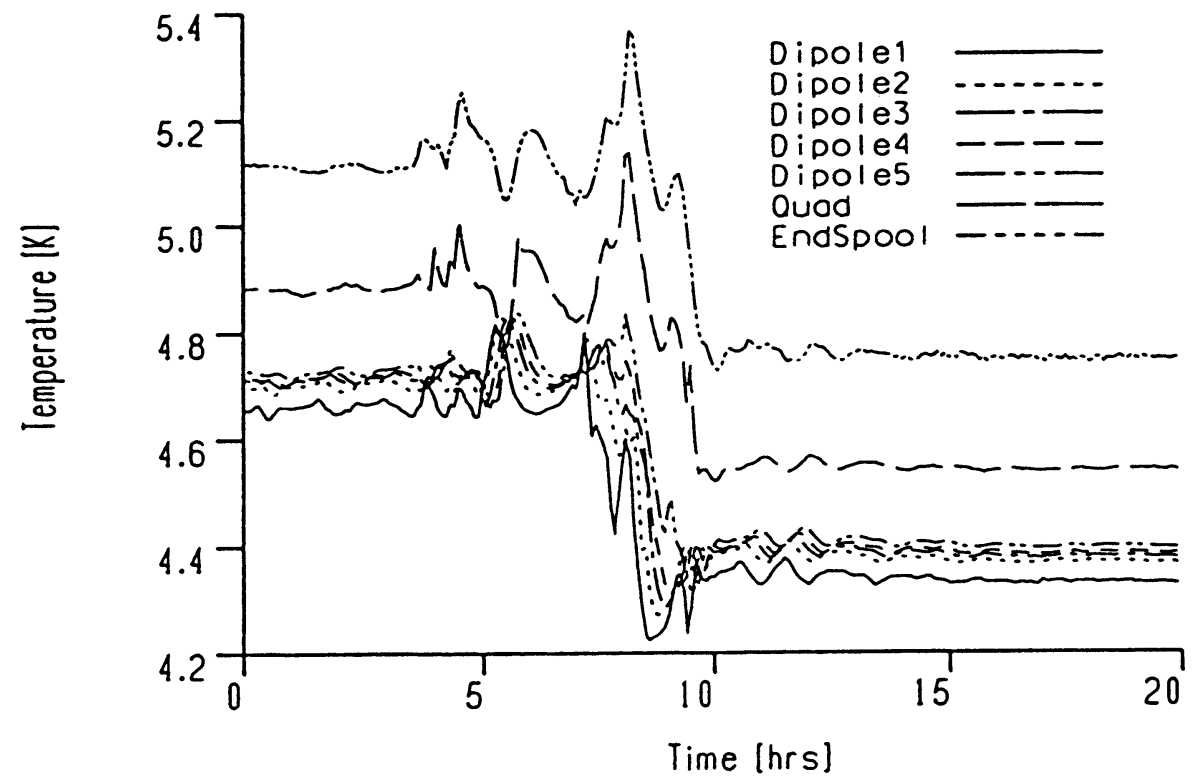

FIGURE 5: An enhanced plot of the low temperature end of the curves shown in Figure 4. This shows the cool down of the magnets to the nominal operating temperature near $4.35 \mathrm{~K}$ after the refrigerator subcooler was activated. The entire string reached is operating temperature in under seven days. The temperature differences between the string components were used to obtain the upper limit estimates of heat leaks into the string elements quoted in the text.

final operating pressure near 4 bars was achieved, the subcooler in the refrigerator was turned on, bringing the dipoles near their operating temperature of $4.3 \mathrm{~K}$, as shown in Figure 5.

The string achieved its operating temperature in less than seven days, which was in agreement with estimates calculated by considering the mass of the magnets and a mass flow rate of $54 \mathrm{~g} / \mathrm{s}$. The cooling waves through each dipole magnet indicated that the dipole magnets behaved similarly and that there were no unusual heat leaks through their cryostats. However, the quadrupole magnet and HSPRE lagged behind the rest of the string by a few tenths of a kelvin, most likely due to a heat leak in the SPR.

After a temperature near $4.3 \mathrm{~K}$ had been reached, the mass flow rates in the power leads were increased to values expected for power operation, about $2 \mathrm{~g} / \mathrm{s}$ each. With these added loads, the refrigeration system still had excess capacity, allowing liquid helium to be produced in the dewar at a rate of $50 \mathrm{l} / \mathrm{hr}$.

\subsection{Preliminary Heat Leak Data}

Measurements were made of the heat leak into the cold mass space and the $80 \mathrm{~K}$ shield. This was done by holding the mass flow constant for a period longer than the 
thermal time constant of the system, measuring the mass flow, and measuring the inlet and outlet temperatures of the fluid flowing through the cold mass and $80 \mathrm{~K}$ shield lines (giving the enthalpy change). Carbon glass resistors with an absolute accuracy of $2.5 \mathrm{mK}$ were used as temperature sensors in the cold mass. Platinum resistors with an accuracy of $\sim 0.5 \mathrm{~K}$ were used as temperature sensors in the $80 \mathrm{~K}$ shield.

In order to reduce end-effects introduced by the presence of the spool pieces, only the heat leaks for the middle three dipoles were considered. The average heat leak for the cold mass for one dipole plus an interconnect was found to be $1.3 \mathrm{~W} \pm 0.4$ $\mathrm{W}$. The uncertainty stems from the absolute accuracy of the temperature sensors. A check on the mass flow measurements was obtained by introducing a known amount of heat into the cold mass via an electric heater, and observing the resulting increase in the heat leak. The measured and known values of the additional heat leak agreed to within $20 \%$. The budgeted heat leak for a cold mass in a dipole magnet plus its interconnect in the collider is about $0.4 \mathrm{~W}$.

The cause for the discrepancies between the measured and budgeted cold mass heat leaks is unknown, although we believe that we have eliminated a poor insulating vacuum as the source. Additional heat leak experiments, including one using a more extensively instrumented dipole magnet, are scheduled for early 1993.

In the same way, the average heat leak to the $80 \mathrm{~K}$ shield as measured. It was found to be $28 \mathrm{~W} \pm 3.9 \mathrm{~W}$. The budgeted heat leak for the $80 \mathrm{~K}$ shield in the collider is $37 \mathrm{~W}$. The heat leak to the $20 \mathrm{~K}$ shield was not measured in this run due to problems with the calibration heater. This measurement will be performed later.

\section{STRING POWER TESTS}

\subsection{Final Electrical Checks}

With the string cold mass near $4.3 \mathrm{~K}$ and filled with helium fluid, the final electrical continuity and hipot tests were performed on the power system, including the power supply and associated instrumentation connected to the power buses. Although the individual components had passed hipots in air to several kilovolts, we observed breakdowns in the string at voltages near $900 \mathrm{~V}$. The cause was subsequently isolated to glow discharge in gaseous helium at the ends of the connectors on the spool pieces where the voltage taps from the magnets made their connections. The voltage taps made connections to the power bus and magnet coils through $200 \Omega, 5 \mathrm{~W}$ resistors mounted inside the end domes of the magnet cold masses. We therefore felt that even though the voltages might go above $900 \mathrm{~V}$ on a voltage tap during a quench, there was no danger of a spark between the magnet coils and ground that could harm the magnets. The worst case would have been a burned resistor in the cold mass, requiring a string warmup for replacement.

Subsequent to the above electrical checks, hipot checks on the system were made at $720 \mathrm{~V}$ before each power test on the string. Leakage currents were about $2 \mathrm{~mA}$ at this voltage due to the impedances in the isolation amplifiers and the voltage-to-frequency converters monitoring the voltage taps. 
TABLE 2: Results of the Induced String Quenches at Various Current Steps

\begin{tabular}{|c|c|c|c|c|c|c|c|}
\hline $\begin{array}{c}\text { Current } \\
\text { (A) }\end{array}$ & $\begin{array}{l}\text { Magnet/ } \\
\text { Heater }^{+}\end{array}$ & $\begin{array}{c}\mathrm{T} 1 \\
(\mathrm{~ms})^{++}\end{array}$ & $\begin{array}{c}\text { MIITs\# } \\
\text { Dipole }\end{array}$ & $\begin{array}{c}\text { MIITs\# } \\
\text { Quad }\end{array}$ & $\begin{array}{c}\text { Max Volt } \\
\text { to Gnd }\end{array}$ & $\begin{array}{c}\text { Max Volt } \\
\text { in Coils }\end{array}$ & Comments \\
\hline 2000 & $\mathrm{D} 1 / 1$ & 167 & 4.7 & & 45 & 33 & Both buses fired* \\
\hline 2000 & $\mathrm{D} 2 / 1$ & 167 & 6.6 & & 29 & 33 & \\
\hline 2000 & D5/1 & 167 & 4.6 & & 42 & 29 & Both buses fired* \\
\hline 2000 & $\mathrm{D} 3 / 2$ & 183 & 4.6 & & 53 & 41 & Both buses fired* \\
\hline 2000 & $\mathrm{D} 4 / 1$ & 167 & 6.6 & & 24 & 22 & \\
\hline 2000 & $\mathrm{Q} 1 / 1$ & 233 & 6.1 & 4.4 & 33 & 25 & \\
\hline 2000 & $\mathrm{D} 1 / 2$ & 150 & 6.8 & & 30 & 33 & \\
\hline 2500 & $\mathrm{D} 4 / 2$ & 133 & 5.9 & 3.2 & 44 & 32 & \\
\hline 2500 & $\mathrm{D} 2 / 2$ & 150 & 6.0 & 2.1 & 61 & 51 & Both buses fired* \\
\hline 2500 & $\mathrm{D} 5 / 2$ & 133 & 5.7 & 2.9 & 97 & 49 & \\
\hline 2500 & $\mathrm{D} 3 / 1$ & 150 & 5.8 & 2.5 & 122 & 65 & Both buses fired* \\
\hline 2500 & $\mathrm{Q} 1 / 2$ & 217 & 4.7 & 2.9 & 61 & 42 & \\
\hline 3000 & $\mathrm{D} 5 / 2$ & 133 & 6.7 & 4.1 & 138 & 86 & \\
\hline 3000 & $\mathrm{D} 1 / 1$ & 133 & 7.3 & & 86 & 74 & \\
\hline 3500 & $\mathrm{D} 3 / 1$ & 117 & 7.4 & & 357 & 216 & \\
\hline 3500 & $\mathrm{D} 5 / 2$ & 117 & 7.9 & 4.2 & 211 & 141 & \\
\hline 4000 & $\mathrm{D} 3 / 1$ & 117 & 8.0 & 3.8 & 531 & 317 & Both buses fired** \\
\hline 4000 & $\mathrm{D} 3 / 1$ & 117 & 8.0 & 4.0 & 522 & 312 & Both buses fired ${ }^{* *}$ \\
\hline 4500 & $\mathrm{D} 3 / 1$ & 100 & 8.7 & 5.3 & 714 & 434 & Both buses fired ${ }^{* *}$ \\
\hline 5000 & $\mathrm{Q} 1 / 1$ & 167 & 7.5 & 4.4 & 296 & 202 & \\
\hline 5500 & Q1/1 & 150 & 8.2 & 4.7 & 337 & 258 & \\
\hline
\end{tabular}

+ Dipole (D) or quadrupole (Q) magnet number and heater circuit (1 or 2) activated to induce the quench. ${ }^{++}$Time from the initiation of the quench until the quench detection system activated proper heaters in the three magnets on the power bus where quench occurred.

\# MIITs are the integral of $I^{2} \mathrm{dt}$ in units of $10^{6}$ in a magnet after a quench has occurred and can be related to the temperature rise in the coils. MIITs limits for the cable used in the magnets in the string are given in Table 1.

*All magnet heaters on hoth buses were activated intentionally after detecting the quench.

${ }^{* *}$ All magnet heaters on both buses activated after detecting the quench due to thermal propagation.

To test the dump circuit, a 500 A current was run through the string and the dump switch was opened to dissipate the current through the dump resistor. The current decreased exponentially with the expected time constant of $24 \mathrm{~s}$, consistent with the inductance of all six of the magnets in the string and the resistance of the dump resistor. 


\subsection{The Power Test Program}

The program to bring the string to full current operation included a sequence of induced quenches at currents of 2000 and $2500 \mathrm{~A}$. In these tests, every pair of heater circuits in each magnet was activated in turn to induce the quench, and the QPM responded by firing the heaters in the magnets sharing the bus where the quench was detected. As the magnets were cryo-stable below $2000 \mathrm{~A}$ and a quench would not propagate, little would have been learned about quench performance below that current. The results of these quenches are given in Table 2. In a few of these induced quenches all of the magnets on both buses were forced to quench instead of just the bus where the quench was detected. Except for the two cases in which the quench was induced in the quadrupole, the heater firing times and maximum voltages generated appear fairly uniform throughout the string.

The MIITs generated in the dipole and quadrupole magnets did not vary significantly from quench to quench in the group of quenches where both buses fired or in the group where only one bus fired. This was taken as evidence that the string components behaved in a uniform manner and that significant retuning of the heater energy needed to initiate a quench in a particular magnet was not warranted. The reason for the difference in the MIITs for the two groups was that at $2000 \mathrm{~A}$ the bypass diodes did not switch into the circuit as quickly as they do at higher currents. This causes the current decay in the magnets to take longer than for the quenches where both buses fired. This can also be seen by comparing the MIITs for the 2500 A quenches with those of the 2000 A quenches where only one bus fired. Because the diodes switched on more quickly at $2500 \mathrm{~A}$, the MIITs were actually less. The heater firing logic was also tested successfully, as the proper QPM response occurred in each case.

The current in the string was raised in $500 \mathrm{~A}$ steps, and at each step, quenches were induced in particular magnets to study the performance of the system and to monitor string parameters. The currents were ramped up at rates varying from 2 to $4 \mathrm{~A} / \mathrm{s}$ The induced quenches continued up to $5500 \mathrm{~A}$, where the peak of the MIITs curve for the dipole magnets was expected. The results are given in Table 2 . To show representative data on the string performance in more detail, we have chosen a quench at $5500 \mathrm{~A}$ where the quench was isolated to the last three magnets in the string. Also, we show the results of a 4500 A test where all six of the magnets experience a quench that appears to have propagated thermally from the first three magnets on one bus to the last three magnets on the next. Finally the results of the ramp-up to $6500 \mathrm{~A}$ are presented.

\subsection{Data for the String Quench at 5500 Amperes}

5.3.1 Electrical Data The quench at 5500 A was initiated in one of the pairs of strip heaters in the quadrupole magnet, and was detected within $20 \mathrm{msec}$ by the QPM, which, in turn, fired all of the heaters in the fourth and fifth dipole magnets, as well as the other set of heaters in the quadrupole magnet. The power supply was turned off and the dump switch opened, placing the dump resistor into the circuit to extract the current still flowing in the first three dipole magnets in the string that remained superconducting throughout the whole quench sequence. A plot of the current dump 


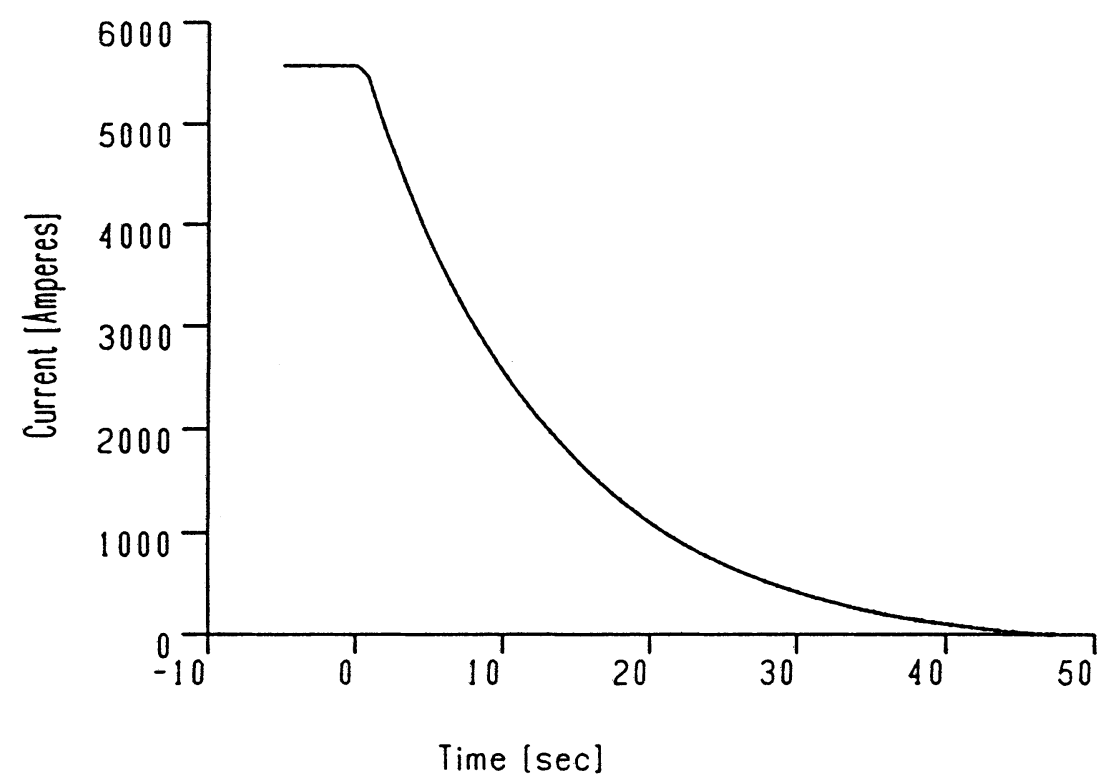

FIGURE 6: The current at the input power bus of the string after the 5500 A quench. The quench was induced in the quadrupole magnet. The current decreases exponentially through the dump resistor with a $12 \mathrm{~s}$ time constant consistent with the inductance of the first three dipole magnets that were still superconducting while the last two dipoles and the quadrupole magnets quenched.

is shown in Figure 6. It exhibited an exponential decrease in current with a $12 \mathrm{~s}$ time constant, consistent with approximately one-half of the inductance of the entire string as opposed to the $24 \mathrm{~s}$ time constant mentioned above for a dump of the entire string.

The observed voltages on the outer coils of the magnets are shown in Figure 7 , where the upper plot is for the first three dipole magnets and the lower plot is for the last two dipole and quadrupole magnets. The sense of the plots is that positive voltages are indicative of resistive voltages (iR), while negative voltages are indicative of inductive voltages $(\mathrm{L} \mathrm{di} / \mathrm{dt}$ ). Knowing the inductances of the magnet coils and calculating di/dt values from the current transductors placed in various parts of the circuit, the inductive contribution to the voltages was subtracted yielding the purely resistive voltages plotted in Figure 8. It is evident from this plot that the first three dipole magnets did not quench. Also, the inductive part of the voltages in the quenching magnets reduced the observed voltages by over a factor of two. The maximum voltage to ground observed in the system during the quench was $296 \mathrm{~V}$ (see Table 2).

The maximum voltages in the quenching magnets were observed in the fifth dipole (DCA316) and measured about $280 \mathrm{~V}$. The largest voltages were always generated in DCA316 regardless of the upper-bus magnet in which a quench was initiated. We also 


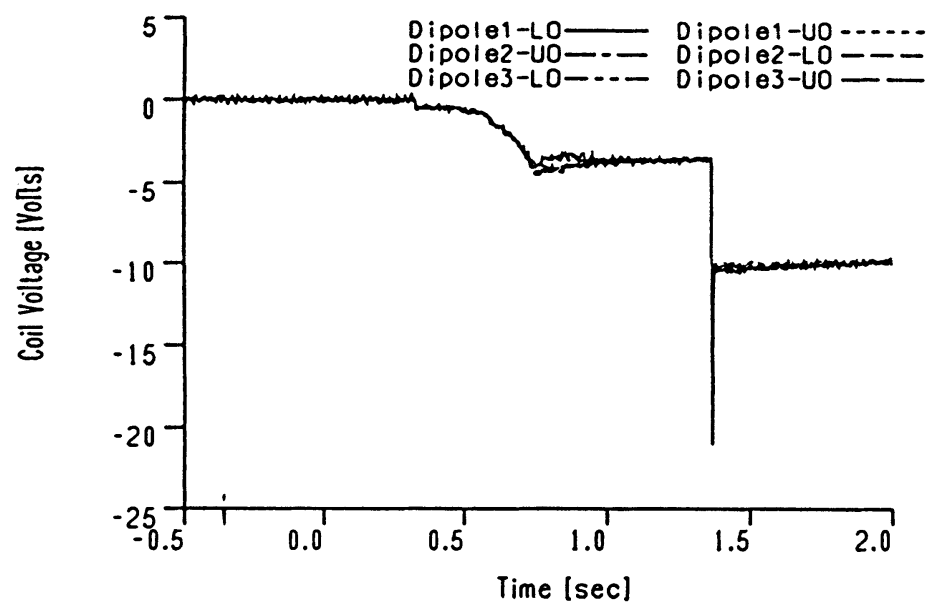

FIGURE 7(a):

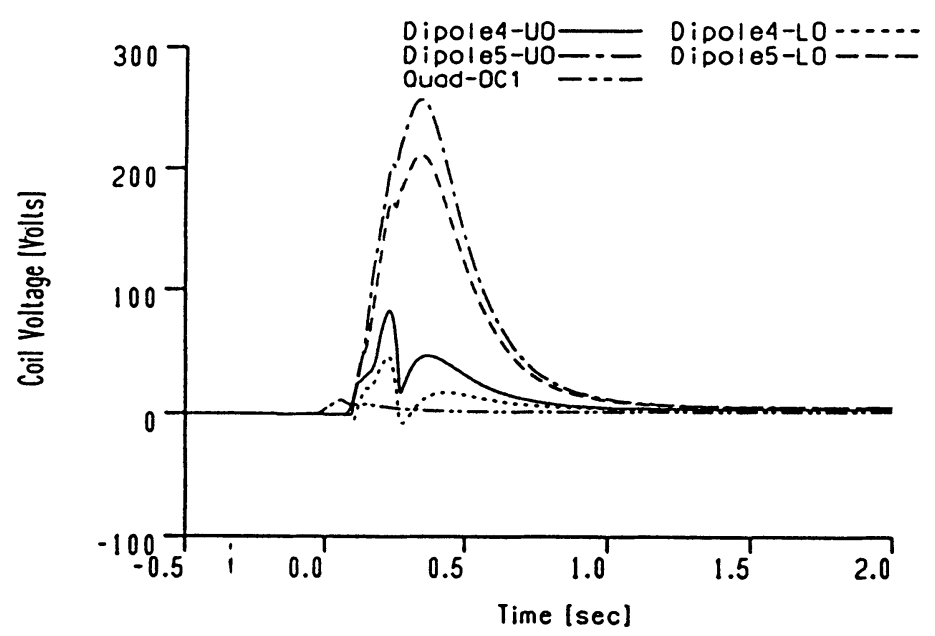

FIGURE 7(b):

FIGURE 7(a,b): (A) The voltage profiles for the outer coils of the first three dipole magnets during the 5500 A quench. These magnets are connected in series on the same power bus. (B) The voltage profiles for the last two dipole and quadrupole magnets connected in series on the other bus. A positive voltage is indicative of resistance (iR) while a negative voltage is indicative of inductance $(\mathrm{L} \mathrm{di} / \mathrm{dt})$. The quench was initiated in the quadrupole magnet. 


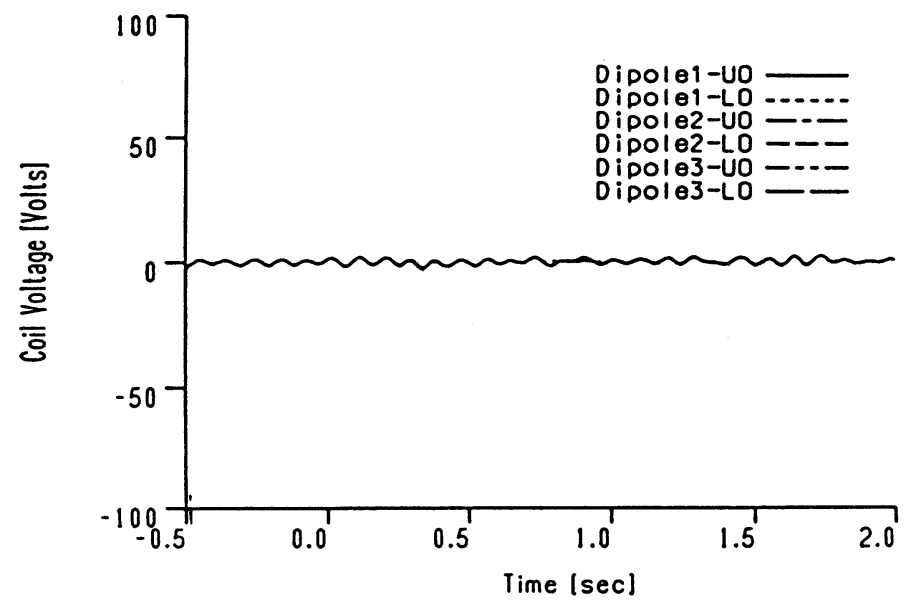

FIGURE 8(a):

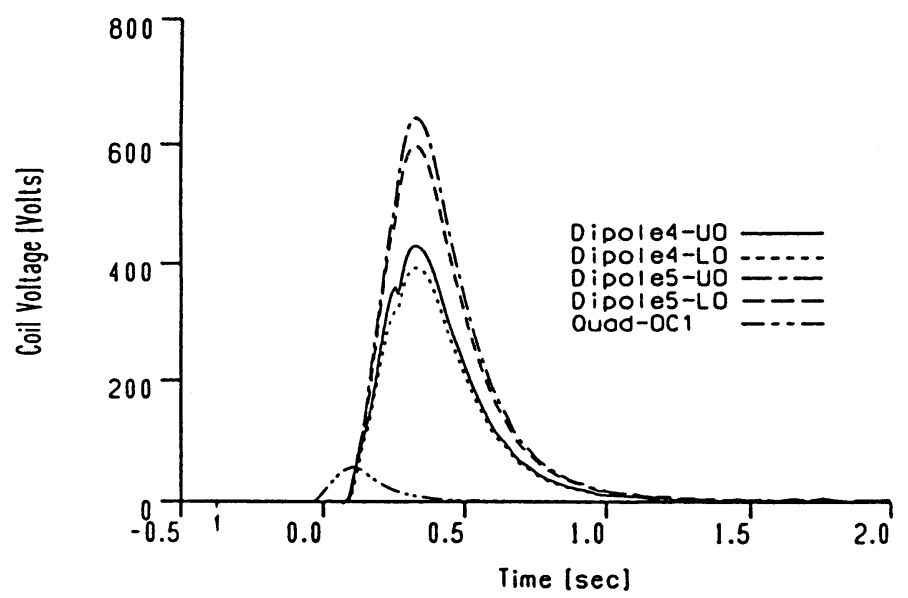

FIGURE 8(b):

FIGURE 8(a,b): (A) The resistive voltage profiles on the outer coils of the first three dipole magnets for the 5500 A quench after the inductive contributions to the voltages were calculated and subtracted. (B) The resistive voltages for the last two dipoles and the quadrupole after the inductive contributions have been subtracted. It is clear that the quench was confined to the last two dipole and quadrupole magnets. 


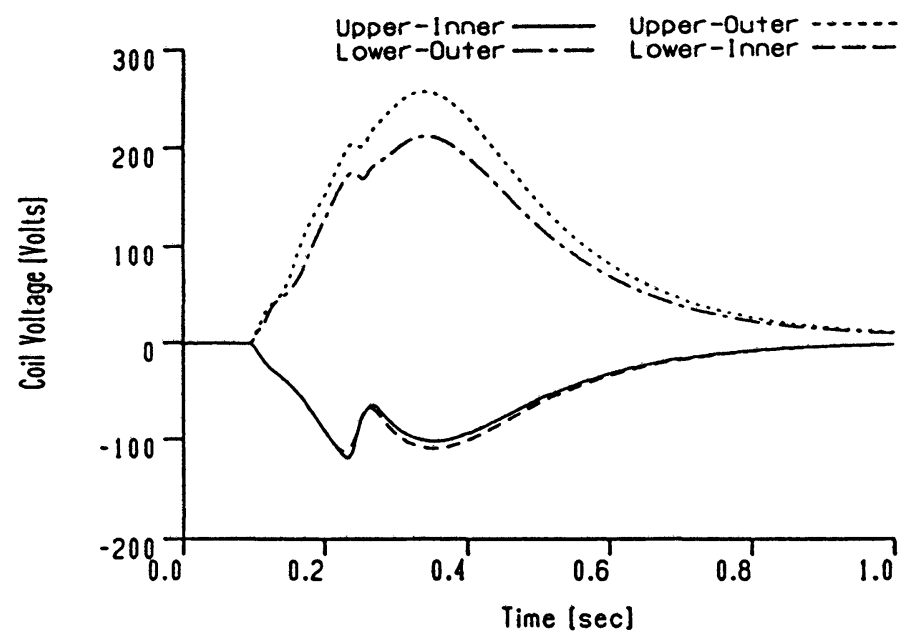

FIGURE 9(a):

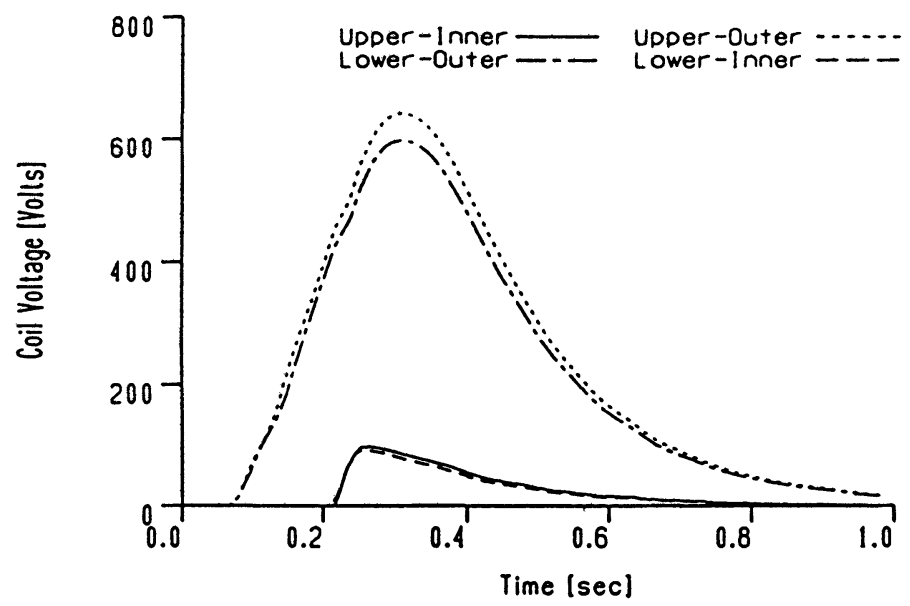

FIGURE 9(b):

FIGURE 9(a,b): (A) The voltage profiles generated in the inner and outer coils of the fifth dipole magnet (DCA316) during the 5500 A quench. (B) The resistive voltages for the four coils shown in Figure 9A after the inductive contributions were subtracted. The quench was initiated in the quadrupole magnet about 40 $\mathrm{ms}$ before time zero. The dipole heaters were fired after the quench was detected. The outer coils were the first to go normal since the heaters were mounted on their outside edge. The heat eventually propagates to the inner coils which go normal beginning about $110 \mathrm{~ms}$ later than the outer coils. By that time the current has decreased significantly, yielding their lower voltages. 


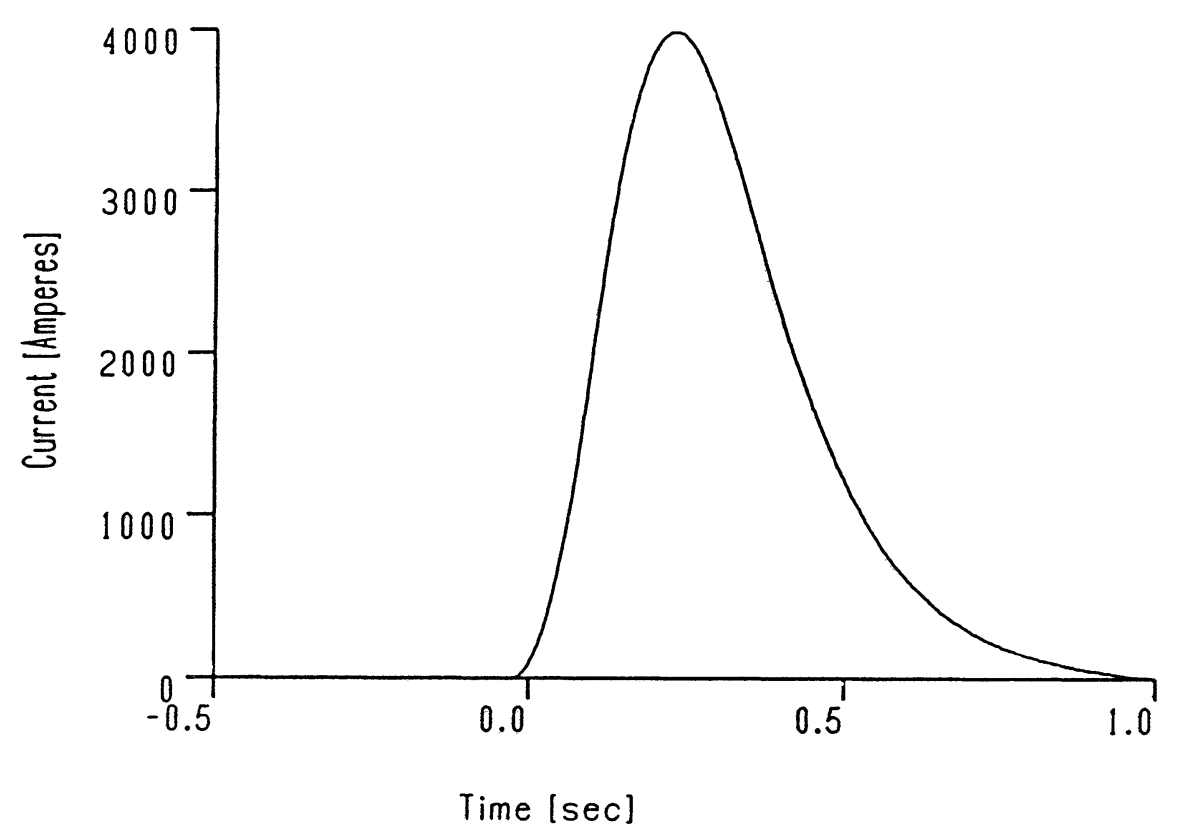

FIGURE 10: The current flowing through the bypass lead in the SPR during the 5500 A quench. The lead provided additional protection for the quadrupole magnet because current from the dipole magnets in series with it was bypassed around the quadrupole. About 2.2 MIITs were dissipated in the stainless steel lead, raising its temperature to $350 \mathrm{~K}$.

observed the highest voltages in magnet DCA319 (the third dipole magnet) regardless of where a quench was initiated on the lower bus (see the following discussion on the quench occurring at $4500 \mathrm{~A}$ ). We believe this was due to the smaller residual resistivity ratio (RRR) ${ }^{20}$ of the copper used in the outer coil cables in these two magnets; the RRR was about half that of the outer coil cable used elsewhere in the string. The smaller ratio implies a higher resistance in the copper that carries the current after the superconducting strands in the cable go normal in a quench, and therefore that higher resistive voltages will be generated for equivalent volumes or lengths of cable.

In Figure 9 we show the voltages for both the inner and outer coils on magnet DCA316. The observed voltages are on the upper plot, and the resistive component of the voltages are on the lower plot. The quench was initiated in the quadrupole about $40 \mathrm{~ms}$ before the time origin of the plot. The origin corresponds to the time when the quench was detected by the QPM and the heaters were activated in DCA316. The outer coil cable starts to go normal about $100 \mathrm{~ms}$ later. The heaters were mounted on the outer surface of the outer coils, and the time needed for the heat to propagate to the inner coils is evident, as they quenched about $120 \mathrm{~ms}$ later - after the current had decayed considerably. Thus, the resistive voltages generated in the inner coils were much less than in the outer coils. 


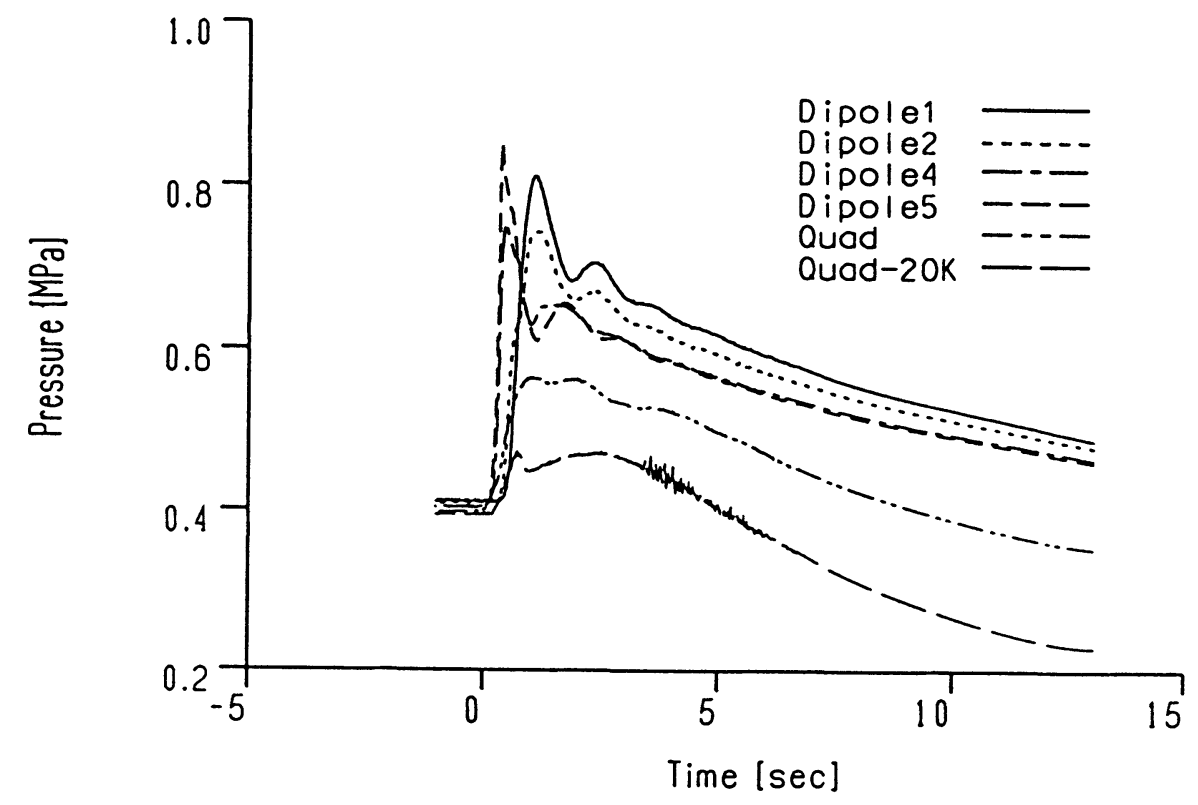

FIGURE 11: The pressures generated in the magnet cold masses during the first seconds after the quench at $5500 \mathrm{~A}$. The decrease in pressures after the spike was due to helium gas escaping through the quench valve which opened pneumatically in the HSPRE for one minute immediately after the quench was detected. The valve allows the gas to flow up the $20 \mathrm{~K}$ shield line back to the refrigerator. The lowest curve shows the pressure in the $20 \mathrm{~K}$ line.

The number of MIITs generated in DCA316 was 7.5 , and in the quadrupole magnet 4.7. This was well below the estimated cable limits given in Table 1 for these magnets. The single magnet test stand data taken for DCA316 showed peaking in the MIIT curve around 5500 to $6000 \mathrm{~A}$, measured as $10.8 .^{21}$ The string test MIITs were below this value as well, which may be explained in part by the higher operating temperatures at which we did our test $(4.6 \mathrm{~K}$ as opposed to the nominal $4.35 \mathrm{~K}$ for the single magnet tests).

In Figure 10 we show the current that flowed through the bypass lead in the SPR during the quench. As mentioned above, this bypass was necessary to provide additional protection for the quadrupole magnet when connected in series with the dipole magnets due to its reduced MIIT capacity. About 2.2 MIITs were generated in the stainless steel lead, raising its temperature to about $350 \mathrm{~K} \cdot{ }^{22}$ After the quench, this heat was removed by flowing helium vapor up the lead for about three minutes.

5.3.2 Cryogenic Data The pressures generated in the string in the first seconds following the $5500 \mathrm{~A}$ magnet quench are shown in Figure 11. The maximum pressure of about $0.83 \mathrm{MPa}$ occurred as a pressure spike just after the quench, and was observed in the interconnect region between the fourth and fifth dipoles. It was caused 


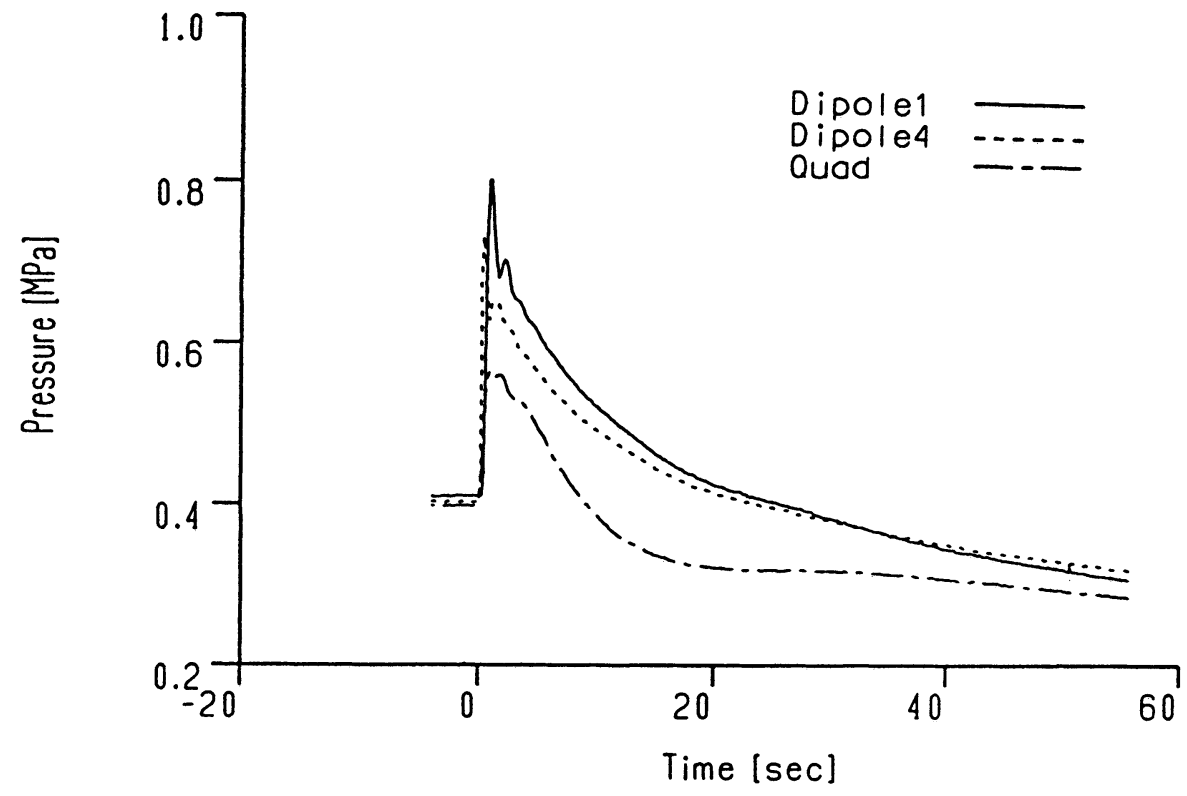

FIGURE 12: Representative string pressures generated in three of the magnet cold masses the first minute after the quench at $5500 \mathrm{~A}$. A thermodynamic model of the magnet quench in reference 19 predicted the second pressure rise occurring about $30 \mathrm{~s}$ after the initial peak as heat propagated through the magnet iron yoke (see the text). There is a small knee evident at this time, though it is more pronounced in the plot in Figure 18 for the $4500 \mathrm{~A}$ quench. The detailed shape of the pressure curve at long times depends on the operating positions of valves in the refrigerator.

by the rapid heating and expelling of the helium vapor from the magnet coils where the quenches occurred, and measured about twice the pressures seen in single magnet test stand data due to the rush of hot gas from the quenching dipoles on either side of the interconnect. The pressure wave propagated throughout the string, arriving at the upstream end in hundreds of milliseconds. The quench valve on the HSPRE was opened pneumatically, allowing the expelled gas to escape the magnet cold mass into the $20 \mathrm{~K}$ shield line and back to the refrigerator: The pressure in the $20 \mathrm{~K}$ shield line upstream of the quench valve is also shown in Figure 11, showing helium vapor flowing less than $0.5 \mathrm{~s}$ after the pressure spike appeared in the cold masses. This valve remained open for one minute after the quench, which resulted in the pressure decay at longer times as shown in Figure 12.

The cold mass temperatures appear in Figure 13. The highest temperatures above $9 \mathrm{~K}$ appear in the quadrupole and the fifth dipole magnets tens of seconds after the quench. This reflects the flow of hot gas down the string toward the quench valve in the HSPRE. After exiting the string, the hot helium gas was diverted to the refrigerator storage dewar. Immediately thereafter the refrigerator began to recool the string, requiring about 3.5 hours to stabilize the string to a temperature at which current 


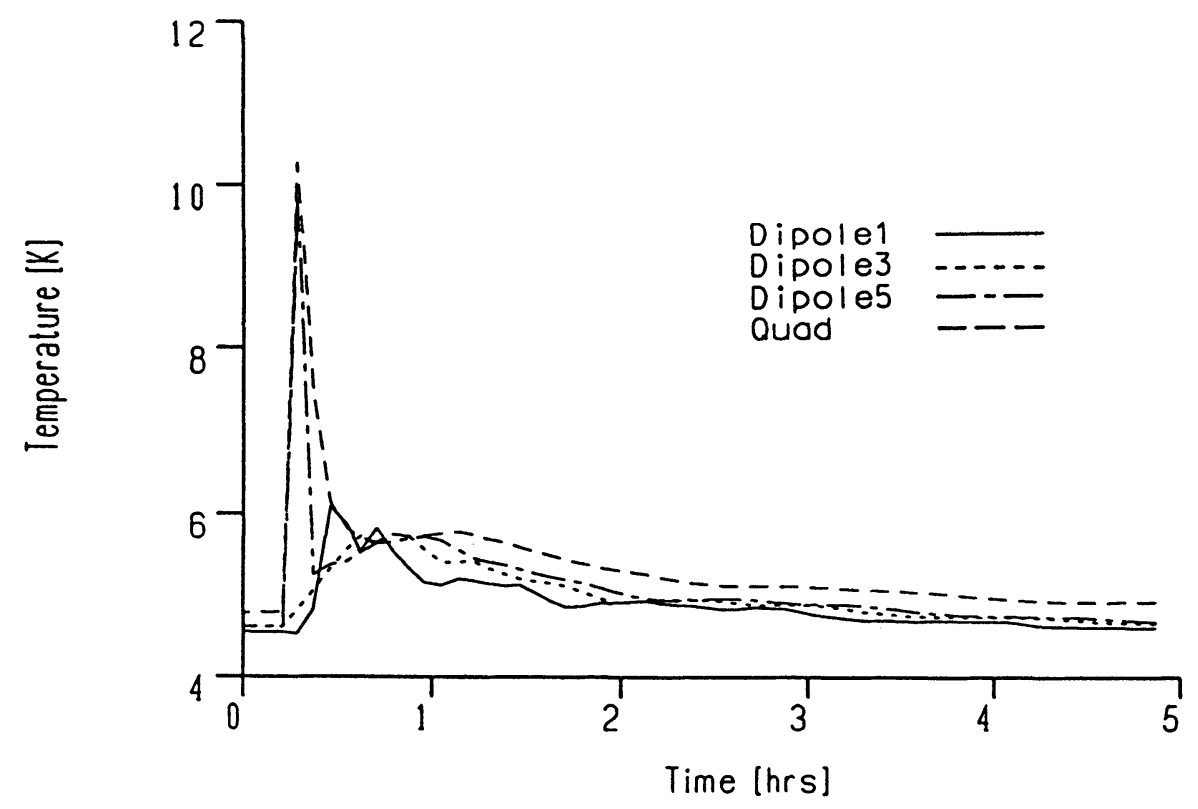

FIGURE 13: The temperatures in four of the magnet cold masses after the 5500 A quench. The maximum helium temperatures were observed in the quadrupole and were due to the flow of the gas toward the quench valve that was opened in the HSPRE just after the quench was detected. The refrigerator recooled the string and stabilized its temperature back at the operating point after $3.5 \mathrm{hrs}$.

could be run again. The recovery process was controlled manually by the refrigerator operators.

The behavior of the cold mass pressures and temperatures was simulated using a model of the magnet assembly and running a thermal dynamics code for the propagation of heat through the cold mass. ${ }^{19}$ The model predicted the pressure spike immediately after the quench and an increase in pressure about $30 \mathrm{~s}$ later. The pressure increase was a result of radial heat propagation outward from the coils, through the stainless steel collars and iron yoke of the magnet, to holes where additional helium fluid was vaporized. The detailed pressure profile depends on the impedances of the cryogenic system, including valve settings and pipe temperatures that can change with time, but the data in Figure 12 show a very subtle knee in the pressure curve near 30 $\mathrm{s}$. This is more noticeable in the data presented in the next section for the $4500 \mathrm{~A}$ test, where all six magnets quenched.

\subsection{Data for the String Quench at 4500 Amperes}

In this test, the quench propagated thermally from the upstream half of the string to the downstream magnets. 


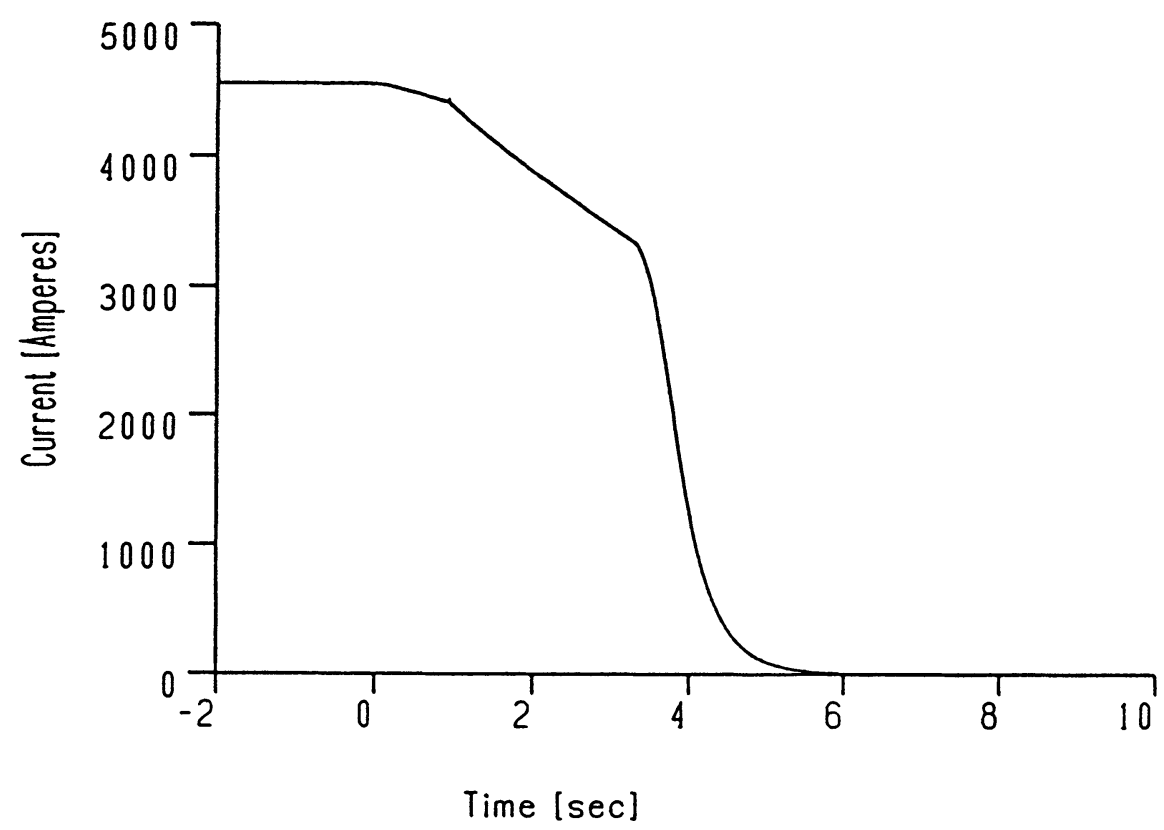

FIGURE 14: The current at the input power bus of the string after the quench at $4500 \mathrm{~A}$. The quench was induced in the third dipole (DCA319). At first the current decreases exponentially through the dump resistor with a $12 \mathrm{~s}$ lifetime indicative of the inductance of the two dipole and quadrupole magnets in the string that remained superconducting while the first three dipole magnets quenched. However, after approximately $4 \mathrm{~s}$, the quench propagates thermally to the fourth dipole magnet, and the last three magnets in the string are quenched thus rapidly reducing the current.

5.4.1 Electrical Data The quench at $4500 \mathrm{~A}$ was initiated in one of the pairs of strip heaters in the third dipole magnet (DCA319) and was quickly detected by the QPM, which fired all the heaters in the first and second dipole magnets as well as the other set of heaters in the third dipole magnet. The power supply was turned off and the dump switch opened, placing the dump resistor into the circuit to extract the current still flowing in the downstream magnets in the string that remained superconducting immediately after the quench began. About $4 \mathrm{~s}$ after the initiation of the quench in the third dipole magnet, the QPM detected a quench in the fourth dipole (DCA315) and fired the heaters in the magnets in the second half of the string. A plot of the current dump is shown in Figure 14. It exhibited an exponential decrease in current with a $12 \mathrm{~s}$ time constant for the first $4 \mathrm{~s}$, then the current decreased rapidly after the last three magnets quenched. Because the second quench occurred so late, we believe it was caused by the thermal propagation of heat released during the quenching of the upstream magnets. This belief is further reinforced by the fact that the first coil to quench in the downstream magnets was a DCA315 inner coil; this should occur when heat propagates via the liquid helium flow path. 


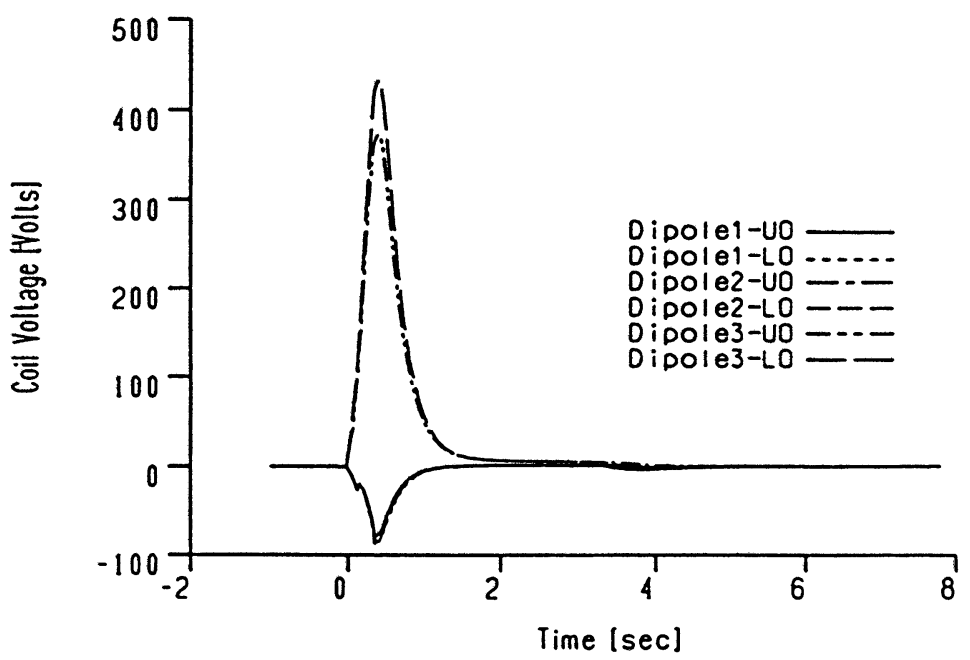

FIGURE 15(a):

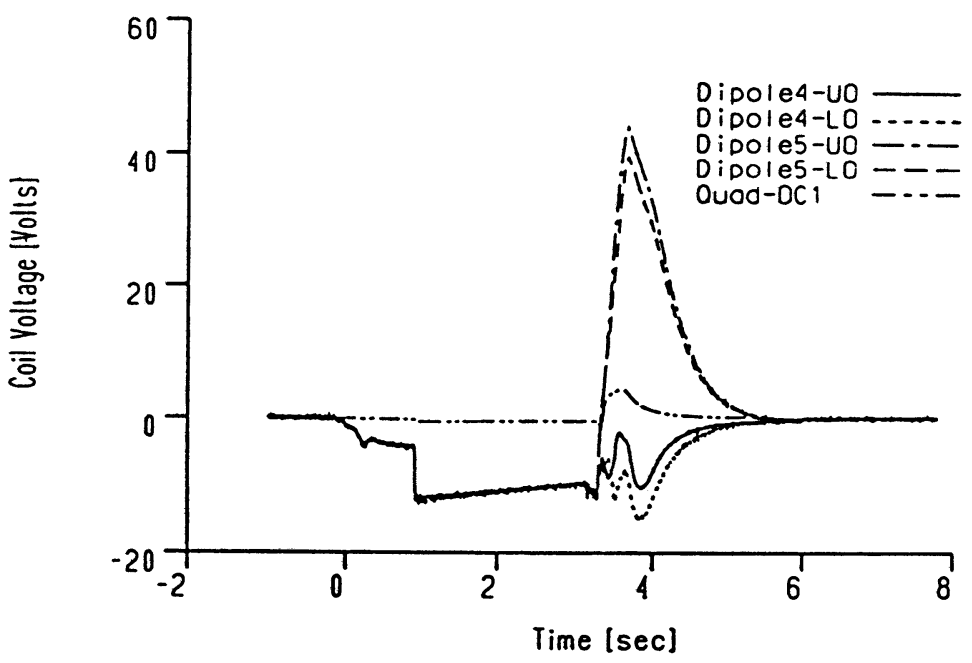

FIGURE 15(b):

FIGURE 15(a,b): (A) The voltage profiles observed for the outer coils of the first three dipole magnets in the string during the 4500 A quench. These magnets are connected in series on the same power bus. (B) The voltage profiles for the last two dipole and quadrupole magnets connected in series on the other bus. A positive voltage is indicative of resistance (iR) while a negative voltage is indicative of inductance ( $\mathrm{Ldi} / \mathrm{dt})$. The quench was initiated in the third dipole magnet. 


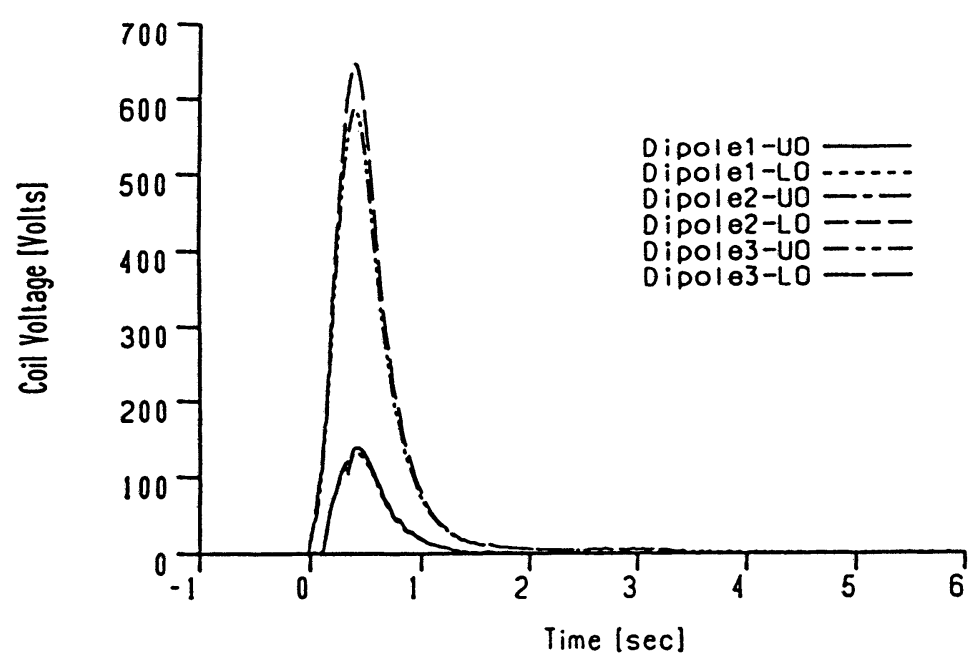

FIGURE 16(a):

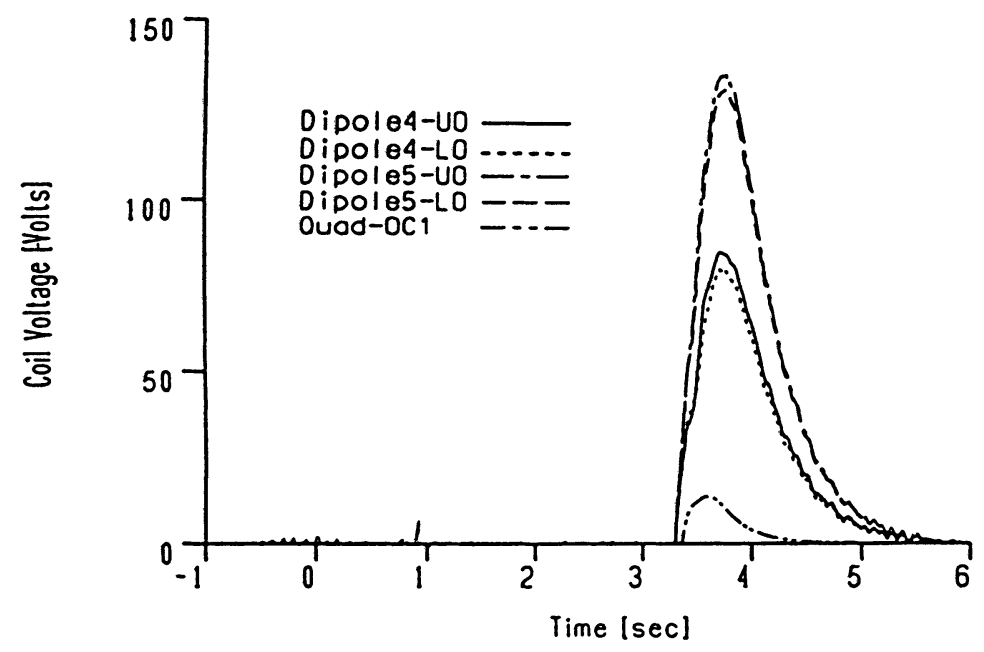

FIGURE 16(b):

FIGURE 16(a,b): (A) The resistive voltage profiles on the outer coils of the magnets for the 4500 A quench after the inductive contributions to the voltages were calculated and subtracted. (B) The resistive voltages for the last two dipoles and the quadrupole. It is clear that the quench was confined to the first three dipole magnets during the first $4 \mathrm{~s}$, and then the last two dipoles and quadrupole magnets quenched. The current was reduced substantially by the time the last three magnets quenched. Therefore, their voltages were less than those of the first three magnets. 


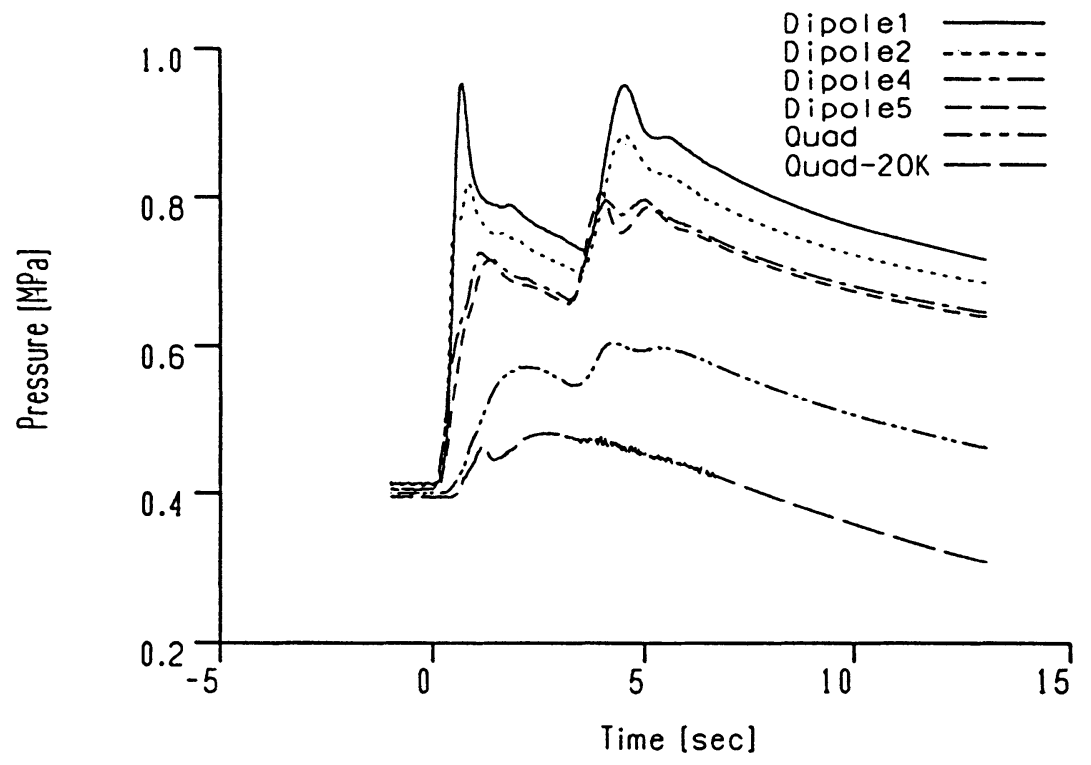

FIGURE 17: The pressures generated in the magnet cold masses during the first few seconds after the quench at $4500 \mathrm{~A}$. The decrease in pressures after the spike was due to helium gas escaping through the quench valve in the HSPRE. The valve was opened pneumatically for three minutes immediately after the quench was detected. The valve allows the gas to flow into the $20 \mathrm{~K}$ shield line and return to the refrigerator. The lowest curve shows the pressure in the $20 \mathrm{~K}$ line. The second pressure spike was due to the quench of the last three magnets in the string as discussed in the text.

The thermal propagation of the quench at $4500 \mathrm{~A}$ was unexpected for the normal operation of the string and we believe that it occurred because of the arrangement where the hot helium gas generated in the quench was exhausted only at the downstream end of the string. This forced the gas to move through the fourth and subsequent magnets on its way back to the refrigeration system. Though a second quench valve was located at the upstream end of the string in the HSPRF design, it was not connected for the initial string tests. We believe that activating this valve - instead of the valve in the HSPRE - during a quench of the first three dipole magnets would have eliminated the thermal propagation of the quench. This is confirmed by the 5500 A data discussed above, where no thermal propagation occurred as the hot gas was exhausted at the appropriate spool to prevent flow to the upstream magnets. The quench valve in the HSPRF will be connected in future power tests.

The observed voltages on the outer coils of the magnets are shown in Figure 15, where the upper plot is for the first three dipole magnets while the lower plot is for the last two dipole and quadrupole magnets. After subtracting the inductive voltages, the purely resistive voltages are plotted in Figure 16. It is evident from this plot that the last three magnets quenched after about $4 \mathrm{~s}$, and that their resistive voltages are considerably less than that observed in the first three dipoles as the current was much reduced at the time of their quench. As mentioned above, the maximum voltages were observed in DCA319. The maximum voltage to ground observed in the system was $714 \mathrm{~V}$. 


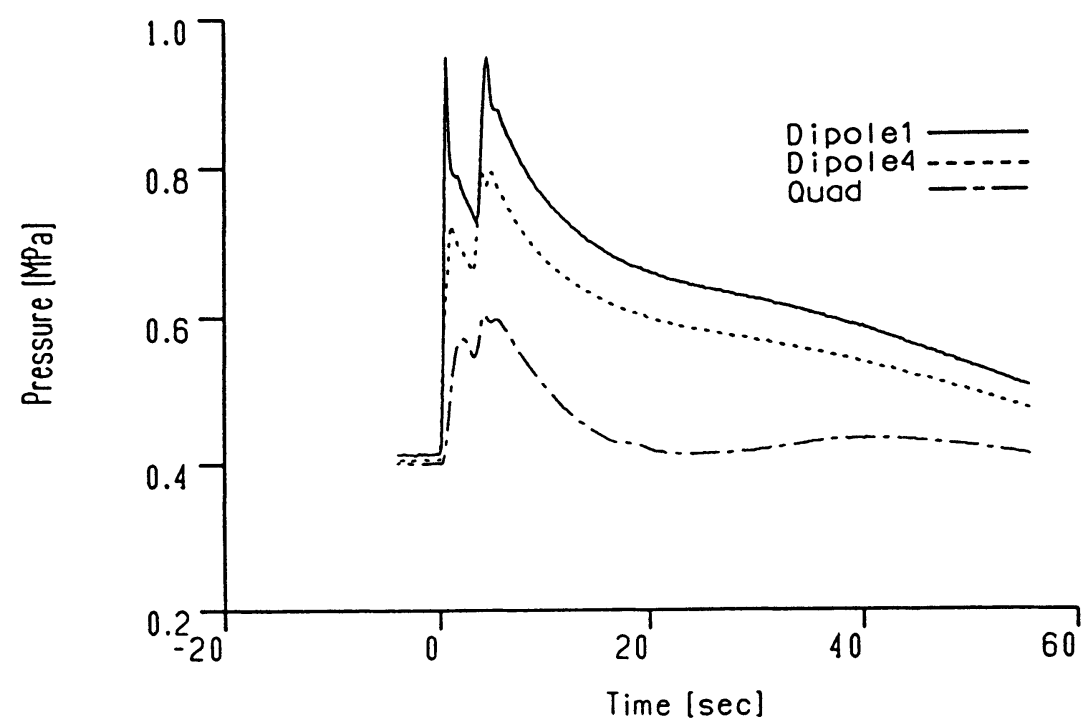

FIGURE 18: Representative string pressures generated in three of the magnet cold masses for the first minute after the quench at $4500 \mathrm{~A}$. A thermodynamic model of the magnet quench in reference 19 predicted the second pressure rise occurring about $30 \mathrm{~s}$ after the initial peak as heat propagated through the magnet iron yoke (see the text). The detailed shape of the pressure curve at long times depends on the operating positions of valves in the refrigerator.

The number of MIITs generated in the third dipole magnet (DCA319) was 8.7. The MIITs in the fourth dipole magnet was 5.3, reflecting that the current had decreased significantly before the quenching of the second half of the magnet string. The single magnet test stand data taken for DCA319 showed a flat distribution in the MIITs curve above $4000 \mathrm{~A}$ and measured 10.0. ${ }^{21}$ The voltages and MIITs were higher in the 4500 A quench than in the 5500 A quench described above. This was due in part to the higher inductance in the first half of the string, where the 4500 A quench was initiated, as compared to the inductance of the second half of the string, which has the lower inductance quadrupole magnet.

5.4.2 Cryogenic Data The pressures generated in the string in the first seconds following the $4500 \mathrm{~A}$ magnet quench are shown in Figure 17. The two pressure spikes corresponding to the quenches in both halves of the string are evident. The maximum pressure of about $0.93 \mathrm{MPa}$ was observed in the interconnect region between the HFSPR and the first dipole magnet. The pressure sensor in the interconnect between the second and third dipole magnets was not working properly and its effects are not plotted in Figure 17. Even though the second pressure spike does arrive at a pressure level similar to the first spike, it should be noted that the cold mass pressures had not been fully relieved through the quench valve by the time of the second quench. The extended time behavior of the cold mass pressures is shown in Figure 18. The knees 


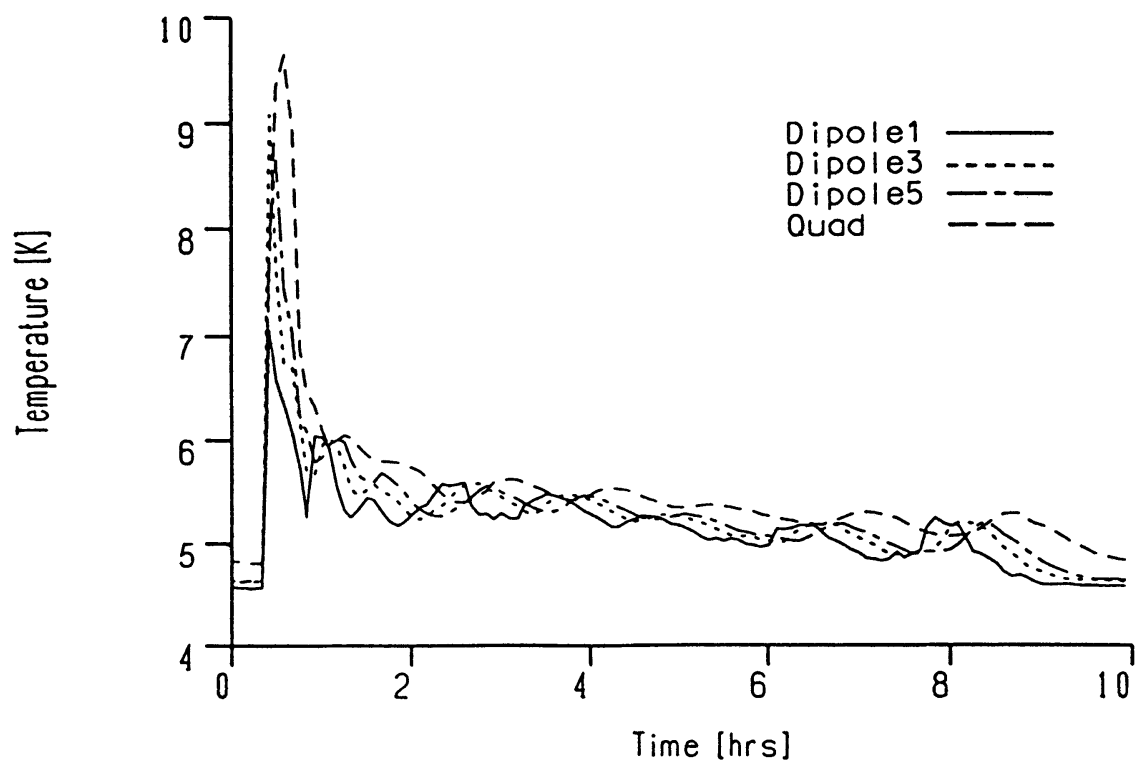

FIGURE 19: The temperatures in four of the magnet cold masses after the 4500 A quench. The maximum temperatures of the helium were observed in the quadrupole. These were due to the flow of the gas toward the quench valve that was opened in the HSPRE just after the quench was detected. The refrigerator recooled the string and stabilized its temperature at the operating point after 10 hours.

in the curves at $30 \mathrm{~s}$ were predicted by the thermodynamic model ${ }^{19}$ and are evident in the data.

The cold mass temperatures appear in Figure 19. The highest temperatures above $9 \mathrm{~K}$ appear in the quadrupole and the fifth dipole magnets more than 30 seconds after the quench. Again this reflects the flow of the hot gas toward the quench valve in the HSPRE. It required about 10 hours to recool the string and stabilize the temperature so that current could be run again. The additional time required to recover after the $4500 \mathrm{~A}$ quench, as opposed to that observed for the $5500 \mathrm{~A}$ quench, was due to the full string quenching.

\subsection{Electrical Data at 6500 Amperes}

The plot of the ramp of the current to $6500 \mathrm{~A}$ is shown in Figure 20. The string was ramped up at $4 \mathrm{~A} / \mathrm{s}$ to $6000 \mathrm{~A}$ and the current held steady for many minutes. During this time, splice joint resistance data were obtained. Then the string was ramped up at $2 \mathrm{~A} / \mathrm{s}$ to a current of $6520 \mathrm{~A}$, a little above the SSC goal of $6500 \mathrm{~A}$. Again the current was held constant for a number of minutes and then the string was ramped down at 4 $\mathrm{A} / \mathrm{s}$. No quenches were encountered during this sequence, and the string components performed as designed. 


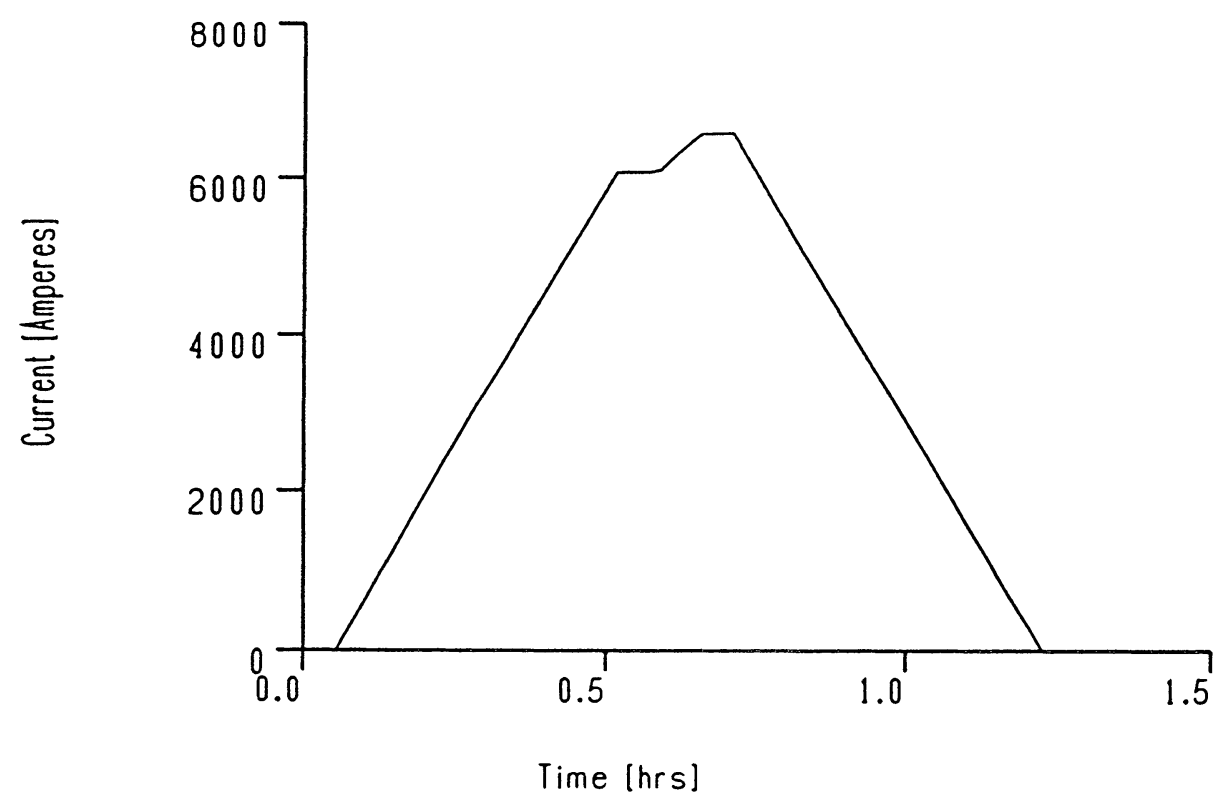

FIGURE 20: The ramp up of the current to the milestone goal is shown, demonstrating full current operation of the string near $6500 \mathrm{~A}$. The current was raised at $4 \mathrm{~A} / \mathrm{s}$ to $6000 \mathrm{~A}$ and the current held constant for many minutes while electrical measurements were made on the string. Then the current was ramped up at $2 \mathrm{~A} / \mathrm{s}$ to $6520 \mathrm{~A}$, just above the current goal, and held there for a number of minutes. The current was then decreased at $4 \mathrm{~A} / \mathrm{s}$. There were no magnet quenches during the ramp up or ramp down sequence.

\section{CONCLUSION}

The Accelerator System String Test successfully achieved the goal of demonstrating full-power operation of SSC prototype dipole and quadrupole magnets arranged in the basic sub-unit of the collider lattice. Extensive data were taken on the electrical and cryogenic performance of the magnets and spool pieces, particularly during magnet quenching. The SSC design concepts for the power and quench protection systems were successfully demonstrated.

In the future, the string will be enlarged to include at least one more half cell of magnets, and the enclosure can ultimately house an additional two half cells on a second tier above the present string. Also, a $6 \mathrm{~kW}$ cryogenics plant designed by Process Systems International ${ }^{23}$ will be part of the final refrigeration for the SSC. Installation will be completed in 1993 and the plant will be commissioned for future use with the ASST. This will permit further study of collider component performance, including detailed heat leak measurements, component reliability testing, and quench propagation from one half cell to another using helium flow rates near $100 \mathrm{~g} / \mathrm{s}$ as anticipated in the collider. 


\section{ACKNOWLEDGMENTS}

The magnets used in the string test were provided by the SSCL Magnet Systems Division (MSD). The magnets were designed, built, individually tested, and delivered to the ASST site by MSD personnel in a cooperative program with their industrial partners and in collaboration with the staff from FNAL, BNL and LBL.

We wish to thank the many technicians that installed and checked the string components under the direction of C. White, M. Hentges and R. Nehring. D. Voy and his software group did an excellent job in writing and implementing the QPS software. The refrigerator crews were under the direction of $\mathrm{H}$. Carter and did a superb job operating a new piece of equipment to achieve the conditions desired for the string.

\section{REFERENCES}

1. J. R. Sanford and D. M. Matthews (eds.), Site-Specific Conceptual Design of the Superconducting Super Collider, Report SSCL-SR-1056 (July 1992).

2. General Dynamics Corporation, Space Systems Division, San Diego, CA.

3. J. Nonte (ed.), in Proceedings of the Third International Industrial Symposium on the Super Collider (Plenum Press, New York, 1991).

4. A. Devred, M. Chapman et al. "Investigation of heater-induced quenches in a full-length SSC R\&D dipole," in Proceedings of the 11th International Conference on Magnet Technology (1992).

5. J. Kuzminski et al, "Quench Performance of 50-mm Aperture 15-m-Long SSCL Dipole Magnets Built at Ferrnilab," to be published in Proceedings of the XVth International Conference on High Energy Accelerators (DESY, Hamburg, Germany, July 1992).

6. C.E. Taylor, C. Peters et al. "Construction and test results on $40-\mathrm{mm}$ bore $5-\mathrm{m}$ long quadrupole magnets for the SSC," in Proceedings of the 1992 Applied Superconductivity Conference (1992).

7. The half feed spool (HFSPR) was manufactured by Meyer Tool Company, Chicago, IL. The collider recooler spool (SPR) was manufactured by CVI, Columbus, OH. The end spool (HSPRE) was manufactured by CKYENCO, Denver, CO.

8. J. Dempko, "Thermal optimization for the helium-cooled power leads for the SSC," (SSCL Technical Report, Preprint \#24).

9. Cryogenics Consultants, Incorporated, Allentown, PA.

10. DYNAPOWER Corporation, Burlington, VT.

11. TISTAR, a product of Siemens Industrial Automation, Inc., Alpharetta, GA.

12. C. Dickey, D. Wallis, J. Weisend II, K. Low, and M. Allen, "A VXI based data acquisition and control system for super collider magnet systems and string tests," Test Engineering Conference Proceedings (Atlanta, GA, June 1991).

13. ORACLE Corporations, Redwood Shores, CA.

14. SUN Microsystems, Mountainview, CA, and Hewlett Packard, Fort Collins, CO.

15. A. McInturff et al, IEEE Trans. Magn. 24, p. 512 (1992).

16. N!POWER, a product of Signal Technology, Inc., Goleta, CA.

17. P. J. Turner, "ACE/gr," Center for Coastal and Land-Margin Research, Oregon Graduate Inst. of Sci. and Tech., Beaverton, OR.

18. R. Parry, "Preliminary design review for the accelerator string test personnel access safety system," AQA-9200009. See also SSCL Technical Report Preprint \#106.

19. R. Carcagno, M. McAshan, and W. Schiesser, "Helium venting model for a SSC half cell," Proceedings of the Third International Industrial Symposium on the Super Collider (Plenum Press, New York, 1991). 
20. The residual resistivity ratio (RRR) is defined as the ratio of the resistivity of a conductor at room temperature to its resistivity at a cold temperature, usually around $10 \mathrm{~K}$.

21. W. Nah, A. Akhmetov, et al., "Quench characteristics of 5-cm aperture 15-m long SSC dipole magnet prototypes," Proceedings of the 1992 Applied Superconductivity Conference (1992).

22. The low number of MIITs generated in the quadrupole during the ASST quenches did not adequately test the need for the bypass circuit that was installed to provide additional protection when the quadrupole was placed in series with the dipole magnets. Though we did not experience any natural quenches in the quadrupole, under natural quenching conditions a larger number of MIITs is expected than we observed in our tests.

23. Process Systems International, Westboro, MA. 